\title{
Arc Measurement of Some Argon Transition Probabilities
}

\author{
C. H. Popenoe and J. B. Shumaker, Jr.
}

(July 29, 1965)

\begin{abstract}
Transition probabilities and line shape parameters for three argon lines have been measured in a wall-stablilized argon arc containing a trace of hydrogen. The $\mathrm{H}_{\beta}$ line shape measurement and the assumption of local thermodynamic equilibrium provided the determination of the argon level populations. From these three lines ten other argon line transition probabilities have been obtained by relative measurements in a pure argon arc. The results are compared with those of other investigators and sources of error are discussed.
\end{abstract}

Key Words: Arc, argon, linewidth, plasma, spectroscopy, stark broadening, transition probability.

\section{Introduction}

The importance of argon transition probabilities for plasma diagnostics is reflected in the large amount of recent experimental work on the measurement of these quantities $[1,2,3,4]{ }^{1} \quad$ The most fruitful source of these measurements has been the high current thermal arc, but unfortunately even here in similar sources the results of different investigators may differ by factors of two, well outside the error estimates attributed to the measurements by their authors. Consequently an additional attempt to measure argon transition probabilities in a thermal arc source was undertaken. The particular technique employed was one which has previously been used in these laboratories [5]. It consists of operating a wall-stabilized arc with a gas mixture containing a trace of hydrogen in the gas whose transition probability is to be measured, and then deducing the arc plasma temperature and composition from the spectral line shape of the $4861 \AA$ hydrogen Balmer $\beta$ line. From the plasma composition and absolute line intensity measurements the transition probabilities can be derived.

Absolute transition probabilities were determined in this manner for the two AI lines at 4300 and $6965 \AA$ and for the AII line at $4806 \AA$. These lines were selected for their prominence, isolation from their neighbors, symmetry, relative freedom from self absorption, and location in wavelength regions favorable for photoelectric detection. Some additional transition probabilities were measured relative to one or another of these three reference lines in pure argon arcs. However, measurements on these lines are less satisfactory either because the lines are weak or because of ambiguity in the location of the background. Most of the prominent blue argon lines in this source are afflicted with this background problem due to the closeness of strong neighbors or asymmetric line

\footnotetext{
${ }^{1}$ Figures in brackets indicate the literature references at the end of this paper
}

shapes (see fig. 1) either of which can result in an uncertainty in the total intensity to be ascribed to the lines.

All of the data recording, handling, and calculations including the graphical presentation of the results were done by automatic machine methods to eliminate as many sources of error as possible. With the statistically large samples made possible by these methods along with elimination of subjective measurements and increased precision, it becomes possible to attempt a more thorough analysis of the errors and uncertainties involved in these measurements.
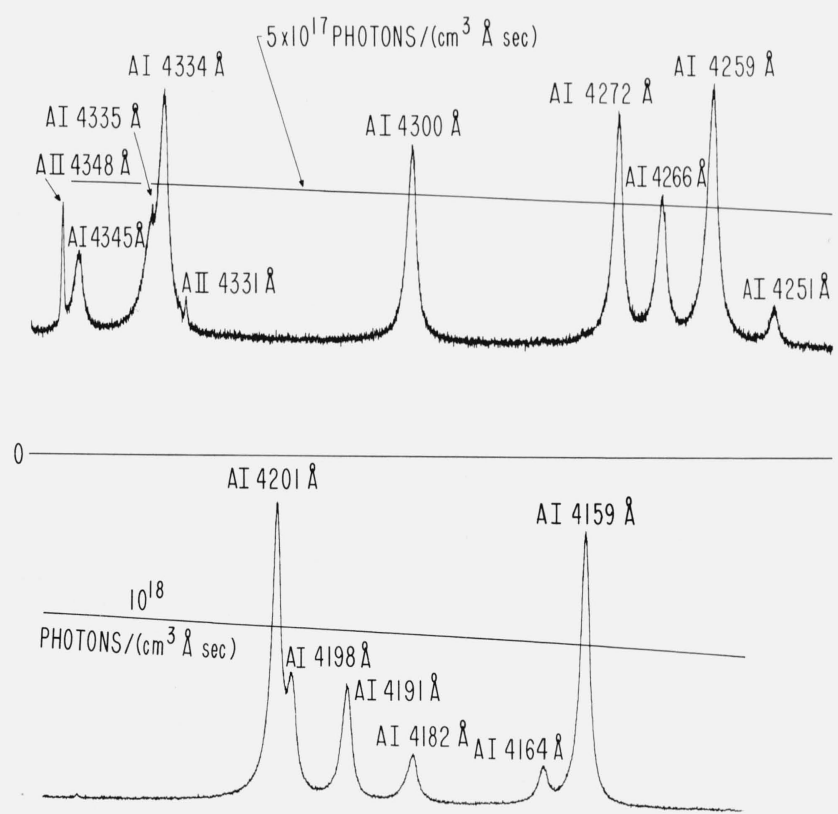

Figure 1. Atmospheric pressure argon arc spectra after Abel inversion [ref. 19].

The total intensity of AI $4300 \AA$ yields an electron density of $6.9 \times 10^{16} \mathrm{~cm}^{-3}$ and tem perature of $12060^{\circ} \mathrm{K}$ assuming the transition probability value of table 3 . 


\section{Experimental Details}

\subsection{Light Source}

The radiation source is a wall-stabilized or cascade d-c arc of about $10 \mathrm{~cm}$ length and $5 \mathrm{~mm}$ diam. The arc chamber is similar to that described in reference [6] with the exception of improved water cooling for higher current operation. The graphite electrodes are surrounded by a separate flow of argon, preventing rapid electrode erosion and contamination of the test gas in the observation section. After passing through the arc the gases are exhausted to the atmosphere through large diameter ports. The arc characteristically operates stably for long periods of time for most test gases. For the experiments reported here arc currents of 40,60, and 90 A were used.

The arc chamber is mounted on a carriage movable by means of a lead screw in a direction transverse to the optic axis of the monochromator. An electrically actuated single-revolution clutch couples an electric motor to the leadscrew drive providing for either continuous or incremental movement of the arc carriage. Variation in rate of scan or increment size is accomplished by a set of quick-change gears in the drive train.

Electrical power is supplied to the arc by either a bank of $1006 \mathrm{~V}$ storage batteries, or a rotary converter of $500 \mathrm{kVA}$ capacity and $650 \mathrm{~V}$ output as shown schematically in figure 2. Both power sources were used during this series of experiments with no observable difference in results. The voltage drop across the arc is typically $102 \mathrm{~V}$ in argon at $60 \mathrm{~A}$, and $112 \mathrm{~V}$ with 1 percent hydrogen added to the test gas. The bulk of the power is dissipated in a large variable water resistor [7] in series with the arc supply.

The current is automatically regulated by a solidstate series current regulator (see fig. 2) consisting of 30 power transistors in parallel, with the current error signal being derived from a $0.2 \Omega$ oil-cooled manganin shunt. The arc current is initially set and later monitored with a potentiometer and galvanometer by which variations in total arc current as small as

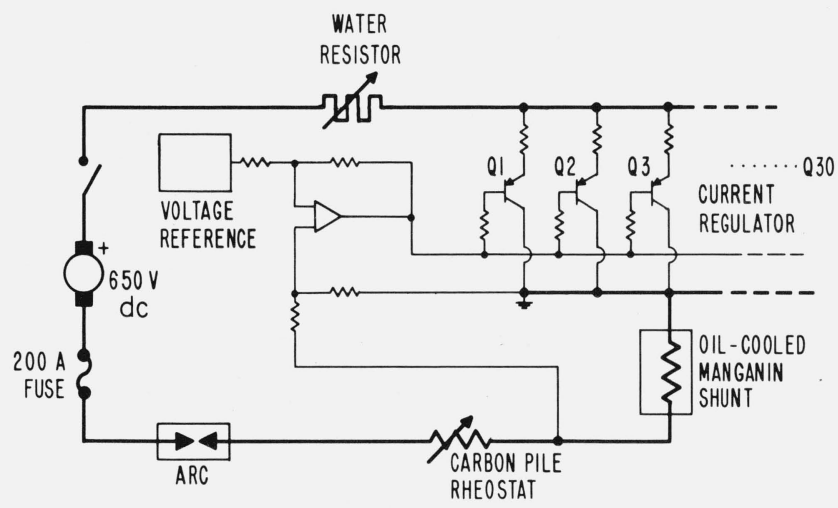

FIGURE 2. Arc circuit schematic including some details of the transistorized automatic current regulator.
$1 \mathrm{~mA}$ may be observed. A carbon pile rheostat is used to provide vernier adjustment of the ballast resistance.

\subsection{Optical and Spectrographic Arrangement}

The arc was viewed in a plane perpendicular to the arc axis with the image focused slightly enlarged on the monochromator entrance slit. A rectangular diaphragm opening at the lens formed the aperture stop of the system and limited the solid angle from the arc to $0.002 \mathrm{sr}$. The dispersing element was an $0.8 \mathrm{~m}$ Ebert-Fastie plane grating monochromator having a nominal dispersion of $10 \AA / \mathrm{mm}$ in the first order. Entrance and exit slits of $15 \mu$ width were used in most of the experiments. The entrance slit height was masked to $1.2 \mathrm{~mm}$ to eliminate reflections from the copper plates adjacent to the observation section of the arc chamber and to minimize the effect of any slight variations of the arc column along its length. During the course of the experiments, RCA 931A (S-4 response), Hamamatsu R-136 and RCA 7265 ( $\mathrm{S}-20$ response), and ITT $\mathrm{FW}-118$ ( $\mathrm{S}-1$ response) photomultiplier tubes were employed to detect the spectral intensity.

The monochromator grating drive is fitted with a single channel paper tape reader attached directly to the wavelength counter to allow direct digital control of the wavelength setting. An electrical signal causes the monochromator to scan in wavelength until a punched hole in the paper tape is sensed by the reader. A program paper tape may therefore be punched to direct the monochromator through a prescribed, not necessarily evenly spaced sequence of wavelengths in the spectrum. The resolution of the tape reader is $0.05 \AA$ and the precision of the wavelength setting better than 0.01 A.

\subsection{Data Recording and Control System}

The large quantity of data encountered in spectrometric studies involving spacial resolution of plasma characteristics invites the use of automatic digital techniques for data collection and reduction [8]. To facilitate data handling the response of the monochromator was recorded digitally on punched paper tape in a form compatible with electronic data processing equipment. In addition, the monochromator wavelength drive and arc carriage positioning mechanism were automatically actuated and coordinated so as to perform the proper variable changes during an experiment in the minimum time.

In essence, the data recorder consists of amplifying, digitizing and tape punching equipment. The small current output of the photomultiplier is amplified and converted to a voltage signal of the order of $1 \mathrm{~V}$ which in turn is converted to a pulse train whose frequency is proportional to this voltage. An electronic counter sums the pulses for a precise interval of time, resulting in a digitized integral of the applied voltage over the counting interval. Upon command, six digits of information from the counter are loaded simultaneously 
into a shift register from which they may be shifted out serially into a code-conversion matrix and punched on paper tape in binary-coded-decimal form.

Movement of the arc carriage, changes in the monochromator wavelength and recording of photomultiplier current are all directed and coordinated automatically during a run by a central programmer. The heart of the programmer is a 25-step, 11 level stepping switch, wired in a closed-loop mode; i.e., a signal from the preceding operation verifying its successful completion is necessary to initiate a succeeding operation. With the above system the rate of data recording is limited by the tape punch to about two 8-character data points per second.

The possibility of erroneous recordings is greatly diminished by various self-checks incorporated into the system. The analog section of the system (current amplifier and voltage-to-frequency converter) is subject to the small errors inherent in analog devices and is therefore checked and calibrated several times during an experiment. The calibration is transferred directly to punched tape to preclude errors in recording. The nature of the digital section, and indeed of digital systems in general is such that the occurrence of small errors in transferring digits through the system is highly improbable. To ensure that such errors, if they occur, will be detected, an odd-bit parity check is made at the tape punch, and the recording process is automatically stopped if an added or dropped bit is detected on the tape. A second and independent parity check is made during the process of converting the tape to punched cards.

\subsection{Experimental Method}

A representative spectral line measurement consisted of the photoelectric recording of the arc intensity at each of perhaps 30 equally spaced positions across the width of the are. This set of measurements was then repeated at each of usually 27 wavelengths spaced nonuniformly across the spectral line-spanning altogether about 10 times the maximum full halfwidth of the line. Finally, without turning off the electronics, recordings were made of the intensity of a calibrated tungsten strip pyrometer lamp at the same 27 wavelengths.

An intermittent arc traversing technique was used in which the motion of the arc carriage was momentarily stopped for an interval of time sufficient to overcome photomultiplier delay effects and to record the intensity at that point before resuming motion and proceeding to the next point. This eliminates the distortion of the arc profile which results from continuous movement of the arc carriage during the counting interval. Also, since the traverses always begin at precisely the same point $( \pm 0.001 \mathrm{~cm})$, each recorded point corresponds to a given point in space referenced to a laboratory coordinate system, not a function of time or scanning rate. The recording positions are therefore repeatable and the recorded intensities directly comparable from traverse to traverse.
The effect of integrating the intensity at a point for a finite interval of time is a temporal smoothing of noise at that point on the arc profile. Some further spacial smoothing occurs because of the necessity of observing a finite "slice" of the arc column. However, the additional loss of spacial resolution which would result from a continuous traversing or "scanning" of the arc image is effectively avoided by the intermittent traversing technique. In these experiments an integrating period of 0.1 sec was used. The width of the observed "slice" was determined by the slit width and in most experiments amounted to about $0.001 \mathrm{~cm}$ while the distance between samples was typically $0.03 \mathrm{~cm}$.

The test gas-either pure argon or premixed mixture of argon and 1 percent hydrogen-was injected tangentially into the arc on both sides of the observation chamber at a rate of 1.2 liter/min and a differential pressure of a few millimeters of water. Gas flow rates were monitored by flowmeters and adjusted to minimize flow in the observation chamber. Separate flows of pure argon at about 0.2 liter/min were injected tangentially at the electrodes. The difference in flow rates insured a net gas flow away from the observation section, further precluding electrode vapor contamination by diffusion.

The intensity measurements are calibrated with a tungsten strip lamp at a brightness temperature at $6530 \AA$ of $2340.3{ }^{\circ} \mathrm{K}$ (IPTS 1948). The lamp was periodically calibrated by the Temperature Physics Section of the National Bureau of Standards. At the conclusion of each arc experiment it was placed at the arc position with the quartz window from the arc placed in the optic path to eliminate the necessity for determination of window transmission. A wellregulated d-c power supply maintained the strip lamp current at a constant level within 0.01 percent. The same punched program tape used to direct the monochromator through the sequence of wavelengths for the experiment was then used to record the calibrated strip lamp intensity at the same wavelengths as those of the experiment.

\section{Treatment of Data}

\subsection{Abel Inversion}

In order to obtain the radial distribution of volume emission in the arc from side-on measurements it is necessary to solve the appropriate Abel integral equation [9] for each traverse across the arc. The technique employed is based upon one described by Freeman and Katz [10] in which the distribution of measured side-on intensities is fitted by a polynomial in the positional coordinate with origin at the center of symmetry. The solution of the integral equation is then obtained analytically for this polynomial and the volume emission at any desired point in the arc is calculated from this solution. By including odd powers in the fitting polynomial some minimal allowance is made for source asymmetry and for errors in locating the position of the arc "center of symmetry" [11]. 
The polynomial fitting was accomplished by the use of the orthogonal properties of the Jacobi [12] polynomials $P_{n}^{(a, a)}(x / R)$ whose inverse Abel transform is expressible in terms of $P_{m}^{(a-1 / 2,0)}\left(2[r / R]^{2}-1\right)$ with odd $n$ terms treated in the approximation of Freeman and Katz [11]. $R$ is the distance from the arc center beyond which the intensity is considered negligible. This technique results in the weighting of the data points by $\left(1-[x / R]^{2}\right)^{a}$. In the experiments reported here uniform weighting $(a=0)$ was arbitrarily used after experiments revealed no appreciable difference in the results obtained using $a=0$ (corresponding essentially to the Freeman and Katz method) and $a=\frac{1}{2}$ (corresponding, except for the odd $n$ terms, to a method described by Herlitz [13]). Individual arc traverses contained from 20 to 41 measurements at equally spaced $x$ points and the resulting intensity profiles were fitted by the Jacobi polynomial expansions with 8 to 12 terms.

\subsection{Line Shape Parameters}

The volume emission intensities calculated from the Abel inversions were reassembled as a function of wavelength at each radial point and a suitable theoretical spectral line shape was fitted by an iterative least squares technique. In the case of the argon lines a dispersion (Lorentz) shape superimposed upon a linear background was used as the fitting function:

$$
\mathrm{I}=A+B\left(\lambda-\lambda_{0}\right)+\frac{C}{1+\left[\left(\lambda-\lambda_{0}\right) / \Delta\right]^{2}}
$$

with $A, B, C, \lambda_{0}$, and $\Delta$ being adjusted to minimize the sum of the squares of the residuals. In the case of the hydrogen $\beta$ lines the line shape fitting was preceded by subtracting from each experimental Abel inverted wavelength scan the corresponding pure argon arc spectrum at the same radial point. This pure argon background spectrum was obtained as a part of each experiment in the same manner and under the same arc conditions as was the argon-hydrogen mixture data. The red and blue wings of the resulting difference spectrum were then averaged and this line shape was fitted to an appropriate theoretical $\mathrm{H}_{\beta}$ line shape:

$$
\mathrm{I}=A+B S(\alpha)
$$

where $\alpha=1.25 \times 10^{-9}\left|\lambda-\lambda_{0}\right|\left(N_{e}\right)^{-2 / 3}, S(\alpha)$ is a theoretical line shape [14], and $A, B$, and the electron density $N_{e}\left(\mathrm{~cm}^{-3}\right)$ are adjusted to minimize the residual function. The Griem, Kolb, and Shen tabulations of $S(\alpha)$ were fitted piecewise by cubics in all the tabulated intervals except the first where a parabola was used with the condition $\frac{d S}{d \alpha}=0$ at $\alpha=0$. The asymptotic wing formulas [15] were used beyond the range of the tables. In this manner piecewise analytic approximations to $S(\alpha)$ were constructed for $\mathrm{H}_{\beta}$. In addition to the dependence of the $\mathrm{H}_{\beta}$ line shapes upon $N_{e}$ implied by the definition of $\alpha$, there is a further weak dependence upon $N_{e}$ and $T$. This dependence was included in these calculations by the actual use of different piecewise line shapes calculated for $N_{e}=10^{17}$ and $T=13000^{\circ} \mathrm{K}, N_{e}=10^{16}$ and $T=10000^{\circ} \mathrm{K}$, and for $N_{e}=10^{15}$ and $T=7500{ }^{\circ} \mathrm{K}$-the first and last being obtained by interpolation in the Griem, Kolb, and Shen tables.

All of the above calculations as well as division by the strip lamp calibration data and conversion to absolute intensities are programmed to be performed in one pass through the IBM 7094 digital computer. At each radial point the computer plots the experimental radially-resolved wavelength scans and tabulates all of the line fitting parameters; in particular the argon line centers $\lambda_{0}, \frac{1}{2}$-halfwidths $\Delta$, and total intensities $\pi C \Delta$, the hydrogen line total intensity $B \int S(\alpha) d \alpha$, and the electron density $N_{e}$.

No slit width corrections were necessary for the hydrogen lines because of their relatively great width. However, for the argon lines slit or apparatus function corrections were required. For this purpose the program for dispersion line shape fitting was systematically calibrated using various ratios of apparatus function width to dispersion line width and using various distributions of wavelength points of measurement across the line. The calibration was done for both Gaussian and triangular apparatus functions and resulted in the application of small slit width dependent corrections to the computed line widths and, for very wide slits, to the line intensities. This procedure should be quite reliable for lines which have nearly a dispersion shape.

The apparatus functions observed with the $15 \mu$ entrance and exit slits used in most of these experiments were approximately Gaussian with $\frac{1}{2}$-halfwidths (one-half of the full width at the half maximum intensity level) of $0.1 \AA$. One experiment was performed with $150 \mu$ slits in which the apparatus function was nearly triangular with a $\frac{1}{2}$-half width of $0.75 \AA$.

Examples of the line shape fitting are given in figures 3 and 4 . The AI $4300 \AA$ line can be seen to exhibit some skewness. This skewness together with the unsymmetric disposition of the wavelength points ${ }^{2}$ causes the dispersion shape fitting routine to produce a line shape which in this example is about 3 percent narrower than a good eye-fit would probably yield. The apparatus function for both lines in this example was the Gaussian shape with $\frac{1}{2}$-halfwidth of $0.1 \AA$. In figure 3 the computer-fitted $\frac{1}{2}$-half width of $0.771 \AA$ required a correction of $0.013 \AA$ for apparatus function resulting in an estimate of $0.758 \AA$ for the $\frac{1}{2}$-halfwidth of the spectral line itself. In figure 4 the intensity scale for $\mathrm{H}_{\beta}$ is displaced vertically by an amount which has no particular physical significance being determined by the subtracted hydrogen-free background spectrum.

Beyond a radial distance of $0.2-.25 \mathrm{~cm}$, the line shape fitting procedure was considered unreliable due to the sharpness of the lines and the attendent small

${ }^{2}$ The asymmetric distribution of wavelength points apparent in figure 3 is, to some extent, unavoidable unless an excessive number of uniformly spaced wavelengths is used because of the dependence of the line shift upon the radial position of the observation. 


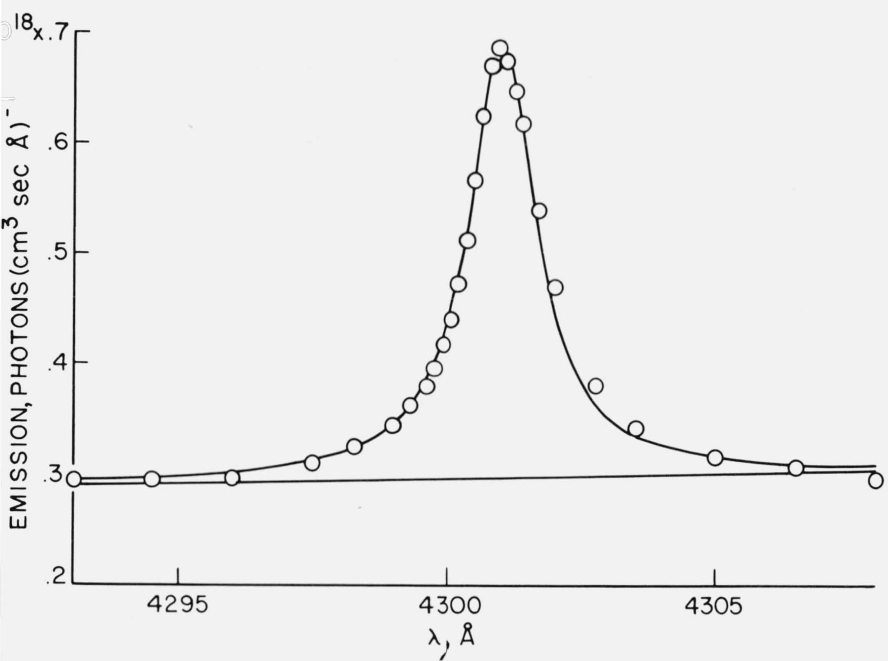

FIGURE 3. Experimental intensity points in the neighborhood of AI $4300 \mathrm{~A}$ after Abel inversion $(0.13 \mathrm{~cm}$ from axis of 90 ampere arc in argon with $\sim 1 \% \mathrm{H}_{2}$ ) showing computer fitted background and dispersion shape: $\frac{1}{2}$-halfwidth $=0.771 \mathrm{~A}$, total line emission $=9.44 \times 10^{17}$ photons $\mathrm{cm}^{-3} \mathrm{sec}^{-1}$

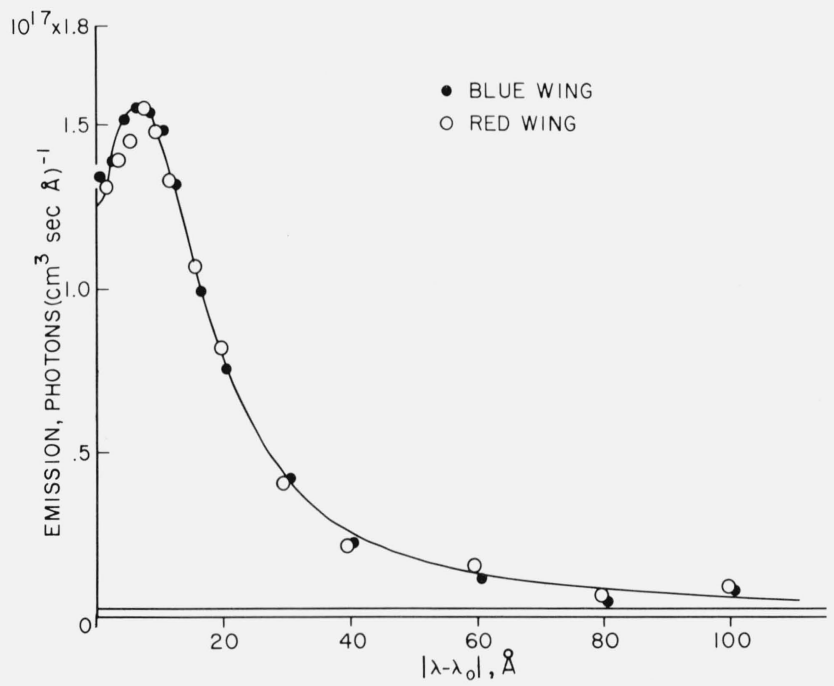

FIGURE 4. Experimental intensity points in the neighborhood of $\mathrm{H}_{\beta} 4861 \AA$ after Abel inversion (same conditions as fig. 3) showing computer fitted background level and theoretical line shape: electron density $=7.45 \times 10^{16} \mathrm{~cm}^{-3}$, total line emission $=8.10 \times 10^{18}$ photons $\mathrm{cm}^{-3} \mathrm{sec}^{-1}$.

number of points defining their shapes. At these distances, the low number density of the emitting species causes a reduction in the signal/noise ratio with the effect that the determination of the true line shape from a statistically small sample of noisy data is improbable. For these reasons, the data from regions lying beyond an arbitrarily chosen radial distance of $0.2 \mathrm{~cm}(0.16 \mathrm{~cm}$ for ionic lines) is not reported here.

Argon line shifts and 1/2-halfwidths corrected for the $0.1 \AA$ Gaussian apparatus function are plotted in

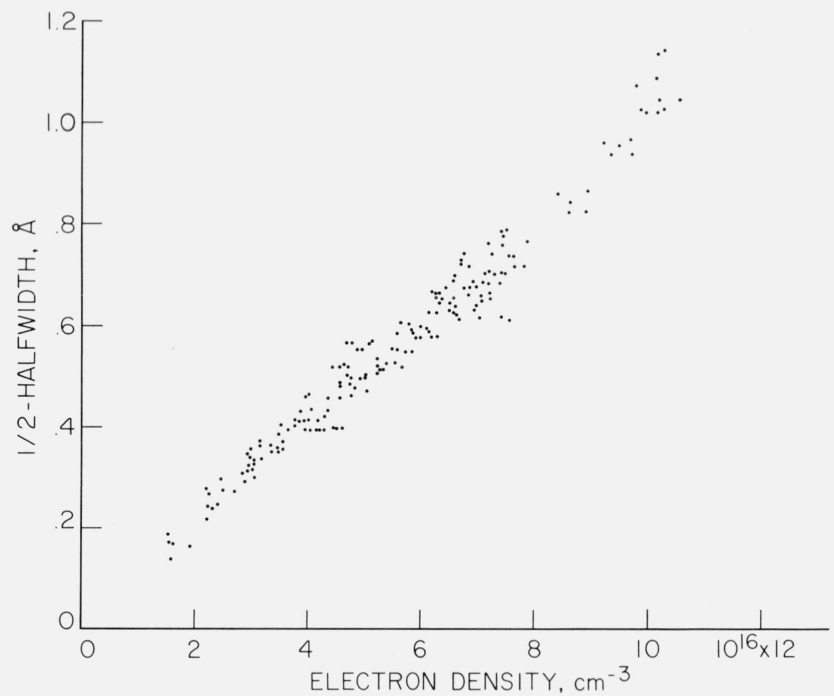

FigURE 5. Dependence of $\frac{1}{2}$-halfwidth upon electron density for AI 4300 A.

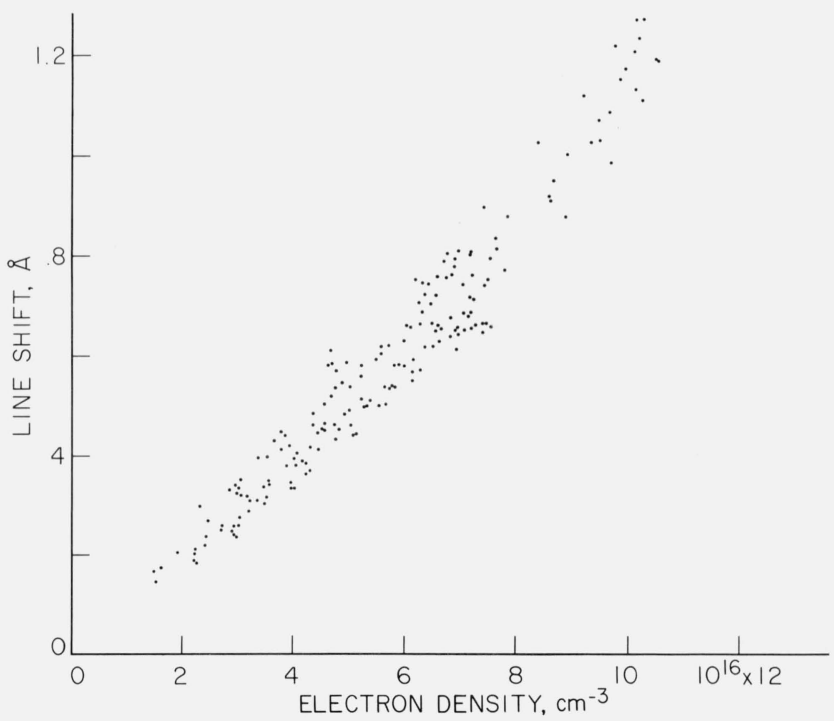

Figure 6. Dependence of the red shift of the AI 4300 A line upon electron density.

figures 5 to 10 against the electron density as determined from the $\mathrm{H}_{\beta}$ line shapes. The wavelengths for the unshifted lines were obtained by extrapolation to $N_{e}=0$. Both the widths and shifts are seen to be approximately proportional to the electron density in rough agreement with theory [9|. The proportionality constants obtained by least squares fitting of this data are shown in table 1 . 


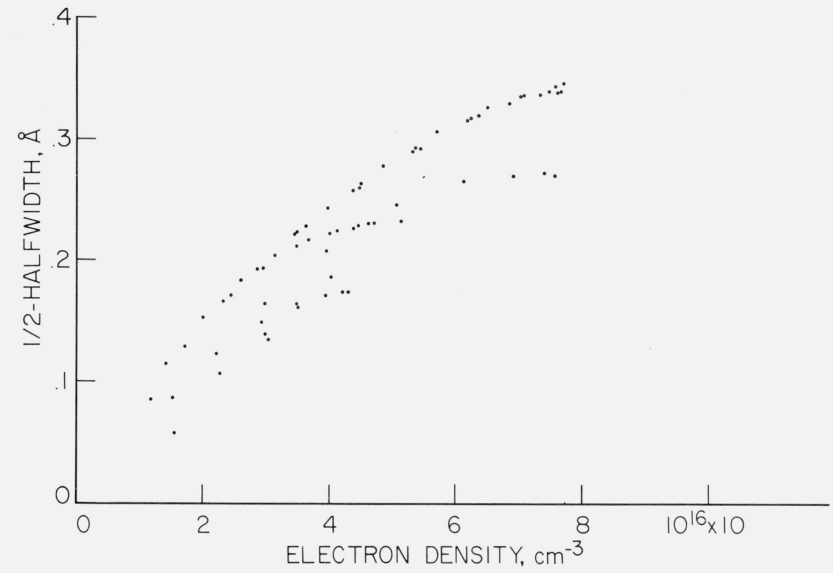

FiguRE 7. Dependence of $\frac{1}{2}$-halfwidth upon electron density for AI 6965 A

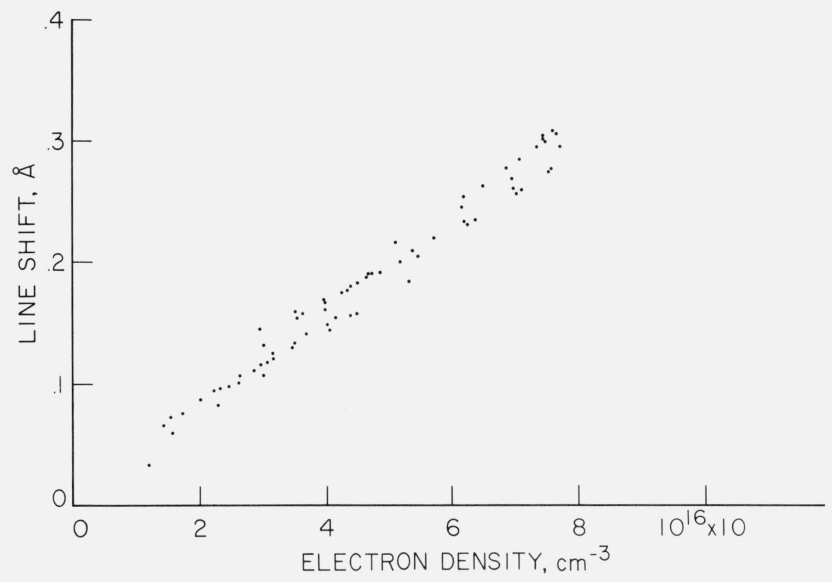

Figure 8. Dependence of the red shift of the AI $6965 \AA$ line upon electron density.

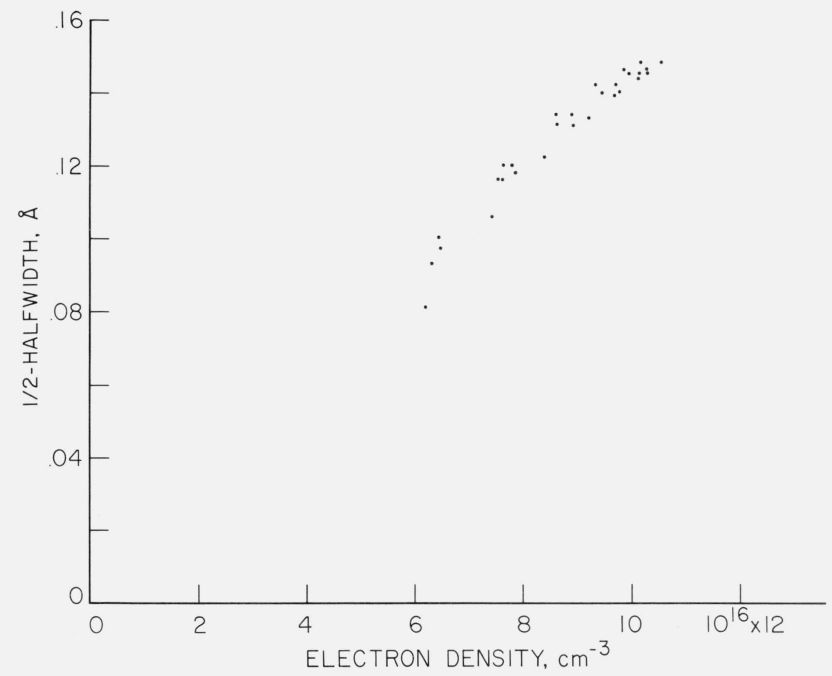

FigURE 9. Dependence of $\frac{1}{2}$-halfwidth upon electron density for AII $4806 \AA$.

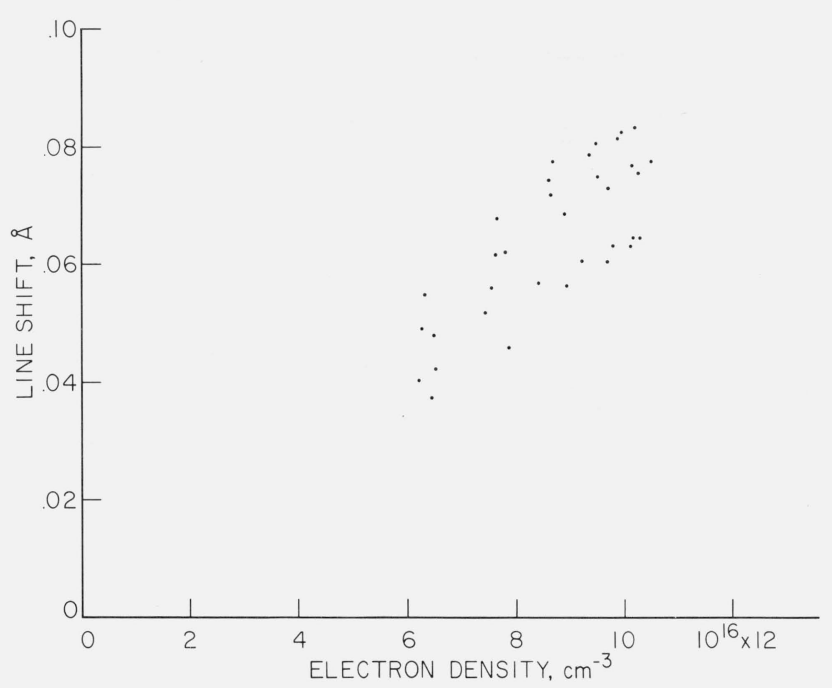

Figure 10. Dependence of the blue shift of the AII $4806 \AA$ line upon electron density.

TABLE 1. Stark 1/2-half widths, w, and shifts, $\mathrm{d}$, for argon lines

\begin{tabular}{l|c|c|c|c}
\hline \multirow{2}{*}{ Line } & \multicolumn{2}{|c|}{$\frac{w}{N_{e}} \times 10^{16}\left[\AA \mathrm{cm}^{3}\right]$} & \multicolumn{2}{c}{$\frac{d}{N_{e}} \times 10^{16}\left[\AA \mathrm{cm}^{3}\right]$} \\
\cline { 2 - 5 } & Expt. & Theor. & Expt. & Theor. \\
\hline & & & & \\
AI $4300 \AA$ & 0.100 & 0.124 & 0.103 & 0.132 \\
AI $6965 \AA$ & .048 & .061 & .039 & .079 \\
AII $4806 \AA$ & .0148 & .0058 & -.0073 & $(-.0022)$ \\
*Shifts to the red are taken as positive.
\end{tabular}

\subsection{Transition Probabilities}

The calculation of the argon transition probabilities requires a knowledge of the plasma composition at each arc data point. For conditions of local thermodynamic equilibrium this can be computed in principle from the total pressure ( $1 \mathrm{~atm})$, measured electron density, and total $\mathrm{H}_{\beta}$ line intensity assuming that only hydrogen and argon are present and using the theoretical $\mathrm{H}_{\beta}$ transition probability, Dalton's law, the condition for quasi-neutrality and the appropariate mass action laws (Saha equations) for the possible ionization and dissociation reactions. The fact that the hydrogen was present in trace amounts permitted some simplification of the calculations as has been discussed previously $[5,16]$. This simplified calculation requires a table of the composition of a pure argon plasma at atmospheric pressure and employs small corrections computed iteratively from the observed $\mathrm{H}_{\beta}$ line intensity.

The pure argon plasma composition was machine computed by a method similar to that of Drellishak, Knopp, and Cambel [17] including first order Debye corrections. The partition functions as functions of the cutoff energy were analytically smoothed and made 
to assume the asymptotic hydrogenlike form at the ionization energy limit (9). The number density of triply ionized argon atoms was assumed negligible thus limiting the calculations to temperatures below about $20000{ }^{\circ} \mathrm{K}$. The computed composition table including upper level populations pertinent to the present experiments is presented as table 2. Griem's treatment [18] of high density corrections to plasma composition calculations suggests that table 2 should be accurate to approximately 1 percent.

Finally the electron density and $\mathrm{H}_{\beta}$ line intensity obtained from the $\mathrm{H}_{\beta}$ line shape fitting are used iteratively in conjunction with this table to determine the total hydrogen content and the number density of excited argon atoms at the radial points considered.
The argon transition probabilities are then found by division of the total line intensities (in photons per cubic centimeter per second) by these number densities at corresponding radii. The individual values of the transition probabilities obtained in this manner are presented in figures 11,12 , and 13 . The averages of these experimental transition probabilities are given in table 3 .

The transition probabilities of the lines other than 6965,4300 , and 4806 were determined relative to one or another of these three reference lines. The lines lying in the red and infrared were measured using the digital apparatus described above with full Abel inversion but with wider slits and with an abbreviated wavelength scan. The plasma composition was

TABLE 2. Argon plasma composition at 1.00 atm pressure*

\begin{tabular}{|c|c|c|c|c|c|c|c|c|c|c|}
\hline$T$ & $Z_{0}$ & $Z_{1}$ & $Z_{2}$ & $U_{0}$ & $U_{1}$ & $U_{2}$ & $N_{e}$ & $N_{0}$ & $N_{1}$ & $N_{2}$ \\
\hline 5000 & 1.0000 & 5.3246 & 7.904 & 127106 & 222812 & $12 \quad 329954$ & $1.322 \mathrm{E} \quad 12$ & $1.468 \mathrm{E} 18$ & 1.322E 12 & $3.642 \mathrm{E}-07$ \\
\hline 5500 & 1.0000 & 5.3752 & 8.034 & 127101 & 222803 & \begin{tabular}{l|l}
03 & 329940
\end{tabular} & $7.174 \mathrm{E} \quad 12$ & $1.335 \mathrm{E} \quad 18$ & $7.174 \mathrm{E} \quad 12$ & $1.441 \mathrm{E}-04$ \\
\hline 6000 & 1.0000 & \multirow{2}{*}{$\begin{array}{l}5.4188 \\
5.4567\end{array}$} & 8.158 & 127093 & 222787 & \begin{tabular}{l|l}
87 & 329916
\end{tabular} & $2.944 \mathrm{E} 13$ & $1.223 \mathrm{E} 18$ & $2.944 \mathrm{E} 13$ & $2.135 \mathrm{E}-02$ \\
\hline 6500 & 1.0000 & & 8.278 & 127081 & 222762 & \begin{tabular}{l|l}
62 & 329879
\end{tabular} & $9.746 \mathrm{E} \quad 13$ & $1.129 \mathrm{E} 18$ & $9.746 \mathrm{E} \quad 13$ & $1.485 \mathrm{E} 00$ \\
\hline 7000 & 1.0000 & 5.4901 & 8.393 & 127063 & 222726 & \begin{tabular}{l|l}
26 & 329825
\end{tabular} & $2.724 \mathrm{E} \quad 14$ & $1.048 \mathrm{E} \quad 18$ & $2.724 \mathrm{E} \quad 14$ & $5.702 \mathrm{E} 01$ \\
\hline 7500 & 1.0000 & 5.5196 & 8.506 & 127039 & $222 t$ & \begin{tabular}{l|l}
78 & 329753
\end{tabular} & $6.648 \mathrm{E} \quad 14$ & $9.775 \mathrm{E} \quad 17$ & $6.648 \mathrm{E} \quad 14$ & $1.361 \mathrm{E} 03$ \\
\hline 8000 & 1.0000 & 5.5459 & 8.615 & 127009 & 2226 & $17 \quad 329662$ & $1.453 \mathrm{E} \quad 15$ & $9.147 \mathrm{E} 17$ & $1.453 \mathrm{E} \quad 15$ & $2.205 \mathrm{E} \quad 04$ \\
\hline 8500 & 1.0000 & 5.56 & 8.722 & 126971 & 2225 & $42 \quad 329549$ & $2.898 \mathrm{E} \quad 15$ & $8.579 \mathrm{E} \quad 17$ & $2.898 \mathrm{E} 15$ & $2.599 \mathrm{E} 05$ \\
\hline 9000 & 1.0001 & 5.59 & 8.827 & 126926 & 222 & \begin{tabular}{l|l}
53 & 329415
\end{tabular} & $5.352 \mathrm{E} \quad 15$ & $8.050 \mathrm{E} 17$ & $5.352 \mathrm{E} \quad 15$ & $2.347 \mathrm{E} 06$ \\
\hline 9500 & 1.0001 & 5.61 & 8.929 & 126875 & 222 & $50 \quad 329261$ & $9.254 \mathrm{E} \quad 15$ & $7.543 \mathrm{E} \quad 17$ & $9.254 \mathrm{E} \quad 15$ & $1.693 \mathrm{E} 07$ \\
\hline 0000 & 1.0003 & 5.62 & 9.029 & 126818 & $222:$ & $35 \quad 329089$ & $1.511 \mathrm{E} 16$ & $7.041 \mathrm{E} 17$ & $1.511 \mathrm{E} \quad 16$ & $1.009 \mathrm{E} 08$ \\
\hline 10500 & 1.0005 & 5.64 & 9.126 & 126755 & 2221 & $09 \quad 328900$ & $2.342 \mathrm{E} \quad 16$ & $6.527 \mathrm{E} 17$ & $2.342 \mathrm{E} \quad 16$ & $5.104 \mathrm{E} 08$ \\
\hline 11000 & 1.0009 & 5.65 & 9.221 & 126688 & 2219 & \begin{tabular}{l|l}
75 & 328699
\end{tabular} & $3.466 \mathrm{E} \quad 16$ & $5.987 \mathrm{E} 17$ & $3.466 \mathrm{E} \quad 16$ & $2.239 \mathrm{E} \quad 09$ \\
\hline .11500 & 1.0015 & 5.67 & 9.314 & 126618 & 2218 & \begin{tabular}{l|l}
36 & 328491
\end{tabular} & $4.910 \mathrm{E} \quad 16$ & $5.412 \mathrm{E} \quad 17$ & $4.910 \mathrm{E} \quad 16$ & $8.668 \mathrm{E} 09$ \\
\hline 12000 & 1.0025 & 5.68 & 9.405 & 126549 & 2216 & \begin{tabular}{l|l}
98 & 328283
\end{tabular} & $6.672 \mathrm{E} \quad 16$ & $4.798 \mathrm{E} \quad 17$ & $6.672 \mathrm{E} \quad 16$ & $3.008 \mathrm{E} \quad 10$ \\
\hline 12500 & 1.0039 & 5.69 & 9.493 & 126482 & 2215 & \begin{tabular}{l|l}
54 & 328082
\end{tabular} & $8.705 \mathrm{E} \quad 16$ & $4.153 \mathrm{E} \quad 17$ & $8.705 \mathrm{E} \quad 16$ & $9.473 \mathrm{E} \quad 10$ \\
\hline 13000 & 1.0061 & 5.70 & 9.579 & 126421 & 2214 & $41 \quad 327898$ & $1.091 \mathrm{E} 17$ & $3.492 \mathrm{E} 17$ & $1.091 \mathrm{E} 17$ & $2.735 \mathrm{E} \quad 11$ \\
\hline 13500 & 1.0093 & 5.71 & 9.663 & 126367 & 2213 & \begin{tabular}{l|l}
34 & 327738
\end{tabular} & $1.315 \mathrm{E} \quad 17$ & $2.843 \mathrm{E} \quad 17$ & $1.315 \mathrm{E} 17$ & 7.307E 11 \\
\hline 14000 & 1.0140 & 5.72 & 9.745 & 126325 & 2212 & $49 \quad 327610$ & $1.525 \mathrm{E} \quad 17$ & $2.235 \mathrm{E} \quad 17$ & $\begin{array}{lll}1.525 \mathrm{E} & 17\end{array}$ & $1.820 \mathrm{E} \quad 12$ \\
\hline 14500 & 1.0208 & 5.73 & 9.825 & 126294 & 2211 & $88 \quad 327517$ & $1.705 \mathrm{E} \quad 17$ & $1.698 \mathrm{E} 17$ & $1.705 \mathrm{E} \quad 17$ & $4.255 \mathrm{E} \quad 12$ \\
\hline 15000 & 1.0304 & 5.74 & 9.902 & 126275 & 2211 & $50 \quad 327462$ & $1.846 \mathrm{E} \quad 17$ & $1.252 \mathrm{E} \quad 17$ & $1.845 \mathrm{E} \quad 17$ & $9.396 \mathrm{E} \quad 12$ \\
\hline 15500 & 1.0441 & 5.75 & 9.978 & 126268 & 2211 & \begin{tabular}{l|l}
35 & 327439
\end{tabular} & $1.942 \mathrm{E} \quad 17$ & $9.030 \mathrm{E} \quad 16$ & $2 \mathrm{E} 17$ & $1.971 \mathrm{E} 13$ \\
\hline 16000 & 1.0630 & 5.75 & 10.052 & 126269 & 2211 & 38 327443 & $1.998 \mathrm{E} \quad 17$ & $6.426 \mathrm{E} \quad 16$ & $1.997 \mathrm{E} \quad 17$ & $3.949 \mathrm{E} \quad 13$ \\
\hline 16500 & 1.0890 & 5.76 & \begin{tabular}{l|l}
62 & 10.124
\end{tabular} & 126277 & 2211 & \begin{tabular}{l|l}
54 & 327467
\end{tabular} & $2.022 \mathrm{E} \quad 17$ & $4.557 \mathrm{E} \quad 16$ & $2.020 \mathrm{E} \quad 17$ & $7.586 \mathrm{E} \quad 13$ \\
\hline 17000 & 1.1241 & 5.77 & 10.195 & 126289 & 2211 & \begin{tabular}{l|l}
78 & 327504
\end{tabular} & $2.021 \mathrm{E} 17$ & $3.248 \mathrm{E} \quad 16$ & E 17 & $1.403 \mathrm{E} \quad 14$ \\
\hline 17500 & 1.1706 & 5.77 & 10.263 & 126304 & 2212 & \begin{tabular}{l|l}
08 & 327549
\end{tabular} & $2.004 \mathrm{E} \quad 17$ & $2.344 \mathrm{E} \quad 16$ & $1.999 \mathrm{E} \quad 17$ & $2.506 \mathrm{E} \quad 14$ \\
\hline 18000 & 1.2315 & 5.78 & 10.330 & 126321 & 2212 & $41 \quad 327598$ & $1.976 \mathrm{E} \quad 17$ & $1.720 \mathrm{E} \quad 16$ & $1.967 \mathrm{E} \quad 17$ & $4.336 \mathrm{E} \quad 14$ \\
\hline 18500 & 1.3099 & 5.79 & 10.395 & 126337 & 2212 & \begin{tabular}{l|l}
75 & 327648
\end{tabular} & $1.943 \mathrm{E} \quad 17$ & $1.288 \mathrm{E} \quad 16$ & $1.928 \mathrm{E} \quad 17$ & $7.283 \mathrm{E} \quad 14$ \\
\hline 19000 & 1.4093 & 5.79 & \begin{tabular}{l|l}
99 & 10.459
\end{tabular} & 126354 & 2213 & \begin{tabular}{l|l}
97 & 327697
\end{tabular} & $1.907 \mathrm{E} \quad 17$ & $9.856 \mathrm{E} \quad 15$ & $1.883 \mathrm{E} 17$ & $1.189 \mathrm{E} \quad 15$ \\
\hline 19500 & 1.5334 & 5.80 & 10.522 & 126369 & 2213 & $38 \quad 327744$ & $1.870 \mathrm{E} \quad 17$ & $7.704 \mathrm{E} \quad 15$ & $1.833 \mathrm{E} \quad 17$ & $1.890 \mathrm{E} \quad 15$ \\
\hline$T$ & $N_{e}$ & & $4044 \mathrm{AI}$ & $4159 \mathrm{~A}$ & & $4259 \mathrm{AI}$ & $4272 \mathrm{AI}$ & $4300 \mathrm{AI}$ & 4348 AII & 4806 AII \\
\hline 5000 & $1.322 \mathrm{E}$ & & 1.153E 04 & $1.669 \mathrm{E}$ & 04 & $2.054 \mathrm{E} \quad 03$ & $1.011 \mathrm{E} 04$ & $1.760 \mathrm{E} 04$ & $4.465 \mathrm{E}-08$ & $6.290 \mathrm{E}-08$ \\
\hline 5500 & $7.174 \mathrm{E}$ & 12 & $2.324 \mathrm{E} 05$ & $3.252 \mathrm{E}$ & 05 & $4.184 \mathrm{E} \quad 04$ & $1.968 \mathrm{E} 05$ & $3.414 \mathrm{E} 05$ & $1.467 \mathrm{E}-05$ & $1.952 \mathrm{E}-05$ \\
\hline 6000 & $2.944 \mathrm{E}$ & 13 & $2.818 \mathrm{E} 06$ & $3.835 \mathrm{E}$ & 06 & $5.118 \mathrm{E} \quad 05$ & $2.319 \mathrm{E} 06$ & $4.009 \mathrm{E} 06$ & $1.840 \mathrm{E}-03$ & $2.333 \mathrm{E}-03$ \\
\hline 6500 & $9.746 \mathrm{E}$ & 13 & $2.313 \mathrm{E} 07$ & $3.073 \mathrm{E}$ & 07 & $4.231 \mathrm{E} 06$ & $1.858 \mathrm{E} 07$ & $3.202 \mathrm{E} \quad 07$ & 1.0 & $1.339 \mathrm{E}-01$ \\
\hline 7000 & $2.724 \mathrm{E}$ & 14 & $1.397 \mathrm{E} 08$ & $1.819 \mathrm{E}$ & 08 & $2.572 \mathrm{E} \quad 07$ & $1.099 \mathrm{E} 08$ & $1.890 \mathrm{E} 08$ & $3.667 \mathrm{E} 00$ & $4.314 \mathrm{E} 00$ \\
\hline 7500 & $6.648 \mathrm{E}$ & 14 & $6.604 \mathrm{E} 08$ & $8.450 \mathrm{E}$ & 08 & $1.223 \mathrm{E} 08$ & $5.102 \mathrm{E} 08$ & $8.755 \mathrm{E} \quad 08$ & 7.675E 01 & $8.764 \mathrm{E} 01$ \\
\hline 8000 & $1.453 \mathrm{E}$ & 15 & 2.557E 09 & $3.222 \mathrm{E}$ & 09 & $58 \mathrm{E} 08$ & $1.945 \mathrm{E} 09$ & $3.331 \mathrm{E} 09$ & $1.100 \mathrm{E} 03$ & $1.223 \mathrm{E} 03$ \\
\hline 8500 & $2.898 \mathrm{E}$ & 15 & $8.398 \mathrm{E} 09$ & $1.044 \mathrm{E}$ & 10 & $1.569 \mathrm{E} 09$ & $6.298 \mathrm{E} 09$ & $1.077 \mathrm{E} \quad 10$ & $1.153 \mathrm{E} 04$ & $1.252 \mathrm{E} 04$ \\
\hline 9000 & $5.352 \mathrm{E}$ & 15 & $2.401 \mathrm{E} 10$ & $2.948 \mathrm{E}$ & 10 & 4.503E 09 & $1.778 \mathrm{E} 10$ & $3.037 \mathrm{E} \quad 10$ & $9.301 \mathrm{E} 04$ & $9.901 \mathrm{E} 04$ \\
\hline 9500 & $9.254 \mathrm{E}$ & 15 & $6.094 \mathrm{E} \quad 10$ & $7.404 \mathrm{E}$ & 10 & $1.147 \mathrm{E} \quad 10$ & $4.464 \mathrm{E} \quad 10$ & $7.614 \mathrm{E} \quad 10$ & $6.016 \mathrm{E} \quad 05$ & $6.288 \mathrm{E} 05$ \\
\hline 10000 & 1. & 16 & $5 E 11$ & & 11 & 10 & 11 & 11 & 06 & \\
\hline 10500 & $2.342 \mathrm{E}$ & 16 & $2.910 \mathrm{E} 11$ & $3.471 \mathrm{E}$ & 11 & $5.509 \mathrm{E} \quad 10$ & $2 \mathrm{E} 11$ & $0 \mathrm{E} 11$ & $1.462 \mathrm{E} \quad 07$ & $1.480 \mathrm{E} 07$ \\
\hline 11000 & $3.466 \mathrm{E}$ & 16 & $5.581 \mathrm{E} \quad 11$ & $6.603 \mathrm{E}$ & 11 & $1.059 \mathrm{E} 11$ & $3.979 \mathrm{E} \quad 11$ & $6.764 \mathrm{E} \quad 11$ & $5.745 \mathrm{E} \quad 07$ & $5.738 \mathrm{E} 07$ \\
\hline 11500 & $4.910 \mathrm{E}$ & 16 & $9.889 \mathrm{E} 11$ & $1.161 \mathrm{E}$ & 12 & $1.881 \mathrm{E} 11$ & $6.997 \mathrm{E} 11$ & $1.189 \mathrm{E} 12$ & $1.985 \mathrm{E} 08$ & $1.958 \mathrm{E} 08$ \\
\hline 12000 & $6.672 \mathrm{E}$ & 16 & $1.624 \mathrm{E} 12$ & $1.895 \mathrm{E}$ & 12 & $3.096 \mathrm{E} 11$ & $1.141 \mathrm{E} 12$ & $1.937 \mathrm{E} 12$ & $6.108 \mathrm{E} 08$ & $5.957 \mathrm{E} 08$ \\
\hline 12500 & $8.705 \mathrm{E}$ & 16 & $2.478 \mathrm{E} \quad 12$ & $2.873 \mathrm{E}$ & 12 & $4.731 \mathrm{E} 11$ & $1.730 \mathrm{E} \quad 12$ & $2.934 \mathrm{E} \quad 12$ & $1.690 \mathrm{E} 09$ & 1.631E 09 \\
\hline 13000 & $1.091 \mathrm{E}$ & 17 & $3.513 \mathrm{E} 12$ & $4.049 \mathrm{E}$ & 12 & $6.719 \mathrm{E} 11$ & $2.438 \mathrm{E} \quad 12$ & $4.133 \mathrm{E} \quad 12$ & $4.242 \mathrm{E} \quad 09$ & $4.055 \mathrm{E} 09$ \\
\hline 13500 & $1.315 \mathrm{E}$ & 17 & $4.631 \mathrm{E} 12$ & $5.311 \mathrm{E}$ & 12 & $8.874 \mathrm{E} 11$ & $3.198 \mathrm{E} 12$ & $5.417 \mathrm{E} \quad 12$ & $9.722 \mathrm{E} \quad 09$ & $9.209 \mathrm{E} 09$ \\
\hline 14000 & $1.525 \mathrm{E}$ & 17 & $5.689 \mathrm{E} \quad 12$ & $6.492 \mathrm{E}$ & 12 & $1.092 \mathrm{E} \quad 12$ & $3.908 \mathrm{E} \quad 12$ & $6.616 \mathrm{E} \quad 12$ & $2.048 \mathrm{E} \quad 10$ & $1.924 \mathrm{E} 10$ \\
\hline 14500 & $1.705 \mathrm{E}$ & 17 & $6.534 \mathrm{E} 12$ & $7.423 \mathrm{E}$ & 12 & $1.256 \mathrm{E} \quad 12$ & $4.468 \mathrm{E} \quad 12$ & $7.560 \mathrm{E} \quad 12$ & $3.991 \mathrm{E} 10$ & $3.720 \mathrm{E} \quad 10$ \\
\hline 1500 & 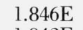 & 17 & E 12 & . & 12 & $1.359 \mathrm{E} 12$ & $4.809 \mathrm{E} \quad 12$ & 2 & 0 & 0 \\
\hline 15500 & $42 \mathrm{E}$ & 17 & $2 \mathrm{E} 12$ & & 12 & E 12 & E 12 & 2E 12 & $1.240 \mathrm{E} \quad 11$ & $1.140 \mathrm{E} \quad 11$ \\
\hline 16000 & $1.998 \mathrm{E}$ & 17 & 7.147E 12 & $8.023 \mathrm{E}$ & 12 & $1.379 \mathrm{E} \quad 12$ & $4.828 \mathrm{E} \quad 12$ & $8.157 \mathrm{E} \quad 12$ & $2.010 \mathrm{E} 11$ & $1.836 \mathrm{E} 11$ \\
\hline 16500 & $2.022 \mathrm{E}$ & 17 & $6.832 \mathrm{E} 12$ & $7.642 \mathrm{E}$ & 12 & 1.319E 12 & $4.599 \mathrm{E} \quad 12$ & $7.767 \mathrm{E} \quad 12$ & $3.116 \mathrm{E} 11$ & $2.829 \mathrm{E} 11$ \\
\hline 17000 & $2.021 \mathrm{E}$ & 17 & $6.394 \mathrm{E} \quad 12$ & $7.128 \mathrm{E}$ & 12 & $1.236 \mathrm{E} \quad 12$ & $4.289 \mathrm{E} \quad 12$ & $7.241 \mathrm{E} 12$ & $4.653 \mathrm{E} 11$ & $4.201 \mathrm{E} 11$ \\
\hline 17500 & $2.004 \mathrm{E}$ & 17 & $5.899 \mathrm{E} \quad 12$ & $6.557 \mathrm{E}$ & 12 & $1.141 \mathrm{E} 12$ & $3.945 \mathrm{E} \quad 12$ & $6.657 \mathrm{E} \quad 12$ & $6.732 \mathrm{E} \quad 11$ & $6.046 \mathrm{E} \quad 11$ \\
\hline 18000 & $1.976 \mathrm{E}$ & 17 & $5.394 \mathrm{E} \quad 12$ & $5.978 \mathrm{E}$ & 12 & $1.045 \mathrm{E} \quad 12$ & $3.596 \mathrm{E} \quad 12$ & $6.067 \mathrm{E} \quad 12$ & $9.479 \mathrm{E} 11$ & $8.470 \mathrm{E} 11$ \\
\hline 18500 & $1.943 \mathrm{E}$ & 17 & $4.905 \mathrm{E} \quad 12$ & $5.421 \mathrm{E}$ & 12 & $9.508 \mathrm{E} 11$ & $3.261 \mathrm{E} 12$ & $5.499 \mathrm{E} \quad 12$ & $1.303 \mathrm{E} \quad 12$ & $1.159 \mathrm{E} \quad 12$ \\
\hline 19000 & $1.907 \mathrm{E}$ & 17 & $4.445 \mathrm{E} \quad 12$ & $4.899 \mathrm{E}$ & 12 & $8.624 \mathrm{E} \quad 11$ & $2.947 \mathrm{E} 12$ & $4.968 \mathrm{E} \quad 12$ & $1.754 \mathrm{E} \quad 12$ & 1.553E 12 \\
\hline 19500 & $1.870 \mathrm{E}$ & 17 & $4.019 \mathrm{E} 12$ & $4.419 \mathrm{E}$ & 12 & $7.804 \mathrm{E} 11$ & $2.658 \mathrm{E} \quad 12$ & $4.480 \mathrm{E} \quad 12$ & $2.313 \mathrm{E} \quad 12$ & $2.039 \mathrm{E} \quad 12$ \\
\hline
\end{tabular}




\begin{tabular}{|c|c|c|c|c|c|c|c|}
\hline$T$ & $N_{e}$ & $6753 \mathrm{AI}$ & $6871 \mathrm{AI}$ & $6965 \mathrm{AI}$ & $7030 \mathrm{AI}$ & $9195 \mathrm{AI}$ & $9292 \mathrm{AI}$ \\
\hline 5000 & $1.322 \mathrm{E} \quad 12$ & $1.016 \mathrm{E} 04$ & $6.565 \mathrm{E} \quad 03$ & $1.626 \mathrm{E} \quad 05$ & $8.131 \mathrm{E} 03$ & $1.891 \mathrm{E} 04$ & $6.510 \mathrm{E} 03$ \\
\hline 5500 & $7.174 \mathrm{E} \quad 12$ & $2.072 \mathrm{E} 05$ & $1.330 \mathrm{E} 05$ & $2.460 \mathrm{E} 06$ & $1.692 \mathrm{E} 05$ & $3.478 \mathrm{E} 05$ & $1.194 \mathrm{E} 05$ \\
\hline 6000 & $2.944 \mathrm{E} 13$ & $2.537 \mathrm{E} 06$ & $1.619 \mathrm{E} 06$ & $2.348 \mathrm{E} \quad 07$ & $2.106 \mathrm{E} 06$ & $3.908 \mathrm{E} 06$ & $1.338 \mathrm{E} 06$ \\
\hline 6500 & $9.746 \mathrm{E} 13$ & $2.099 \mathrm{E} 07$ & $1.333 \mathrm{E} \quad 07$ & $1.574 \mathrm{E} 08$ & $1.768 \mathrm{E} 07$ & $3.007 \mathrm{E} 07$ & $1.028 \mathrm{E} 07$ \\
\hline 7000 & $2.724 \mathrm{E} 14$ & $1.277 \mathrm{E} 08$ & $8.074 \mathrm{E} \quad 07$ & 7.994E 08 & $1.088 \mathrm{E} 08$ & $1.719 \mathrm{E} 08$ & $5.863 \mathrm{E} \quad 07$ \\
\hline 7500 & $6.648 \mathrm{E} 14$ & $6.072 \mathrm{E} \quad 08$ & $3.826 \mathrm{E} 08$ & $3.251 \mathrm{E} \quad 09$ & $5.232 \mathrm{E} 08$ & $7.745 \mathrm{E} 08$ & $2.638 \mathrm{E} 08$ \\
\hline 8000 & $1.453 \mathrm{E} \quad 15$ & $2.364 \mathrm{E} \quad 09$ & $1.485 \mathrm{E} 09$ & $1.104 \mathrm{E} \quad 10$ & $2.056 \mathrm{E} 09$ & $2.876 \mathrm{E} 09$ & $9.785 \mathrm{E} 08$ \\
\hline 8500 & $2.898 \mathrm{E} \quad 15$ & $7.798 \mathrm{E} 09$ & $4.886 \mathrm{E} 09$ & $3.228 \mathrm{E} \quad 10$ & $6.838 \mathrm{E} 09$ & $9.103 \mathrm{E} 09$ & $3.093 \mathrm{E} 09$ \\
\hline 9000 & $5.352 \mathrm{E} \quad 15$ & $2.238 \mathrm{E} 10$ & $1.399 \mathrm{E} \quad 10$ & $8.323 \mathrm{E} \quad 10$ & $1.977 \mathrm{E} 10$ & $2.518 \mathrm{E} 10$ & $8.546 \mathrm{E} 09$ \\
\hline 9500 & $9.254 \mathrm{E} \quad 15$ & $5.703 \mathrm{E} \quad 10$ & $3.557 \mathrm{E} \quad 10$ & $1.926 \mathrm{E} 11$ & $5.071 \mathrm{E} \quad 10$ & $6.207 \mathrm{E} \quad 10$ & $2.105 \mathrm{E} \quad 10$ \\
\hline 10000 & $1.511 \mathrm{E} 16$ & $1.310 \mathrm{E} 11$ & $8.153 \mathrm{E} \quad 10$ & $4.058 \mathrm{E} \quad 11$ & $1.171 \mathrm{E} 11$ & $1.384 \mathrm{E} 11$ & $4.687 \mathrm{E} \quad 10$ \\
\hline 10500 & $2.342 \mathrm{E} \quad 16$ & $2.741 \mathrm{E} 11$ & $1.703 \mathrm{E} 11$ & $7.853 \mathrm{E} 11$ & $2.464 \mathrm{E} 11$ & $2.819 \mathrm{E} 11$ & $9.542 \mathrm{E} \quad 10$ \\
\hline 11000 & $3.466 \mathrm{E} \quad 16$ & $5.270 \mathrm{E} \quad 11$ & $3.270 \mathrm{E} 11$ & $1.407 \mathrm{E} \quad 12$ & $4.762 \mathrm{E} 11$ & $5.289 \mathrm{E} 11$ & $1.789 \mathrm{E} 11$ \\
\hline 11500 & $4.910 \mathrm{E} \quad 16$ & $9.362 \mathrm{E} 11$ & $5.800 \mathrm{E} 11$ & $2.341 \mathrm{E} 12$ & $8.496 \mathrm{E} \quad 11$ & $9.186 \mathrm{E} 11$ & $3.106 \mathrm{E} \quad 11$ \\
\hline 12000 & $6.672 \mathrm{E} 16$ & $1.541 \mathrm{E} 12$ & $9.536 \mathrm{E} 11$ & $3.632 \mathrm{E} 12$ & $1.404 \mathrm{E} 12$ & $1.482 \mathrm{E} 12$ & $5.006 \mathrm{E} 11$ \\
\hline 12500 & $8.705 \mathrm{E} \quad 16$ & $2.356 \mathrm{E} \quad 12$ & $1.456 \mathrm{E} 12$ & $5.256 \mathrm{E} \quad 12$ & $2.155 \mathrm{E} 12$ & $2.223 \mathrm{E} 12$ & $7.505 \mathrm{E} \quad 11$ \\
\hline 13000 & $1.091 \mathrm{E} 17$ & $3.346 \mathrm{E} \quad 12$ & $2.066 \mathrm{E} \quad 12$ & $7.098 \mathrm{E} \quad 12$ & $3.071 \mathrm{E} 12$ & $3.102 \mathrm{E} 12$ & $1.047 \mathrm{E} 12$ \\
\hline 13500 & $1.315 \mathrm{E} \quad 17$ & $4.420 \mathrm{E} \quad 12$ & $2.725 \mathrm{E} \quad 12$ & $8.948 \mathrm{E} 12$ & $4.069 \mathrm{E} 12$ & $4.032 \mathrm{E} \quad 12$ & $\begin{array}{lll}1.360 \mathrm{E} & 12\end{array}$ \\
\hline 14000 & $1.525 \mathrm{E} 17$ & $5.438 \mathrm{E} \quad 12$ & $3.350 \mathrm{E} \quad 12$ & $1.054 \mathrm{E} 13$ & $5.022 \mathrm{E} \quad 12$ & $4.888 \mathrm{E} \quad 12$ & 1.648E 12 \\
\hline 14500 & $1.705 \mathrm{E} 17$ & $6.256 \mathrm{E} \quad 12$ & $3.850 \mathrm{E} \quad 12$ & $1.165 \mathrm{E} 13$ & $5.793 \mathrm{E} 12$ & $5.545 \mathrm{E} \quad 12$ & $1.869 \mathrm{E} 12$ \\
\hline 15000 & $1.846 \mathrm{E} \quad 17$ & $6.773 \mathrm{E} \quad 12$ & $4.165 \mathrm{E} \quad 12$ & $1.214 \mathrm{E} 13$ & $6.287 \mathrm{E} 12$ & $5.925 \mathrm{E} \quad 12$ & $1.997 \mathrm{E} \quad 12$ \\
\hline 15500 & $1.942 \mathrm{E} \quad 17$ & $6.963 \mathrm{E} \quad 12$ & $4.278 \mathrm{E} \quad 12$ & $1.205 \mathrm{E} \quad 13$ & $6.479 \mathrm{E} 12$ & $6.018 \mathrm{E} \quad 12$ & $2.027 \mathrm{E} \quad 12$ \\
\hline 16000 & $1.998 \mathrm{E} 17$ & $6.871 \mathrm{E} \quad 12$ & $4.219 \mathrm{E} \quad 12$ & $1.150 \mathrm{E} \quad 13$ & $6.408 \mathrm{E} \quad 12$ & $5.871 \mathrm{E} \quad 12$ & $1.977 \mathrm{E} \quad 12$ \\
\hline 16500 & $2.022 \mathrm{E} \quad 17$ & $6.576 \mathrm{E} \quad 12$ & $4.035 \mathrm{E} \quad 12$ & $1.067 \mathrm{E} \quad 13$ & $6.146 \mathrm{E} 12$ & $5.559 \mathrm{E} 12$ & $1.872 \mathrm{E} \quad 12$ \\
\hline 17000 & $2.021 \mathrm{E} 17$ & $6.161 \mathrm{E} 12$ & $3.778 \mathrm{E} \quad 12$ & $9.710 \mathrm{E} \quad 12$ & $5.770 \mathrm{E} 12$ & $5.156 \mathrm{E} 12$ & $1.735 \mathrm{E} \quad 12$ \\
\hline 17500 & $2.004 \mathrm{E} \quad 17$ & $5.691 \mathrm{E} 12$ & $3.487 \mathrm{E} 12$ & $8.724 \mathrm{E} \quad 12$ & $5.339 \mathrm{E} 12$ & $4.717 \mathrm{E} \quad 12$ & $1.587 \mathrm{E} \quad 12$ \\
\hline 18000 & $1.976 \mathrm{E} \quad 17$ & $5.209 \mathrm{E} \quad 12$ & $3.190 \mathrm{E} 12$ & $7.780 \mathrm{E} \quad 12$ & $4.895 \mathrm{E} \quad 12$ & $4.279 \mathrm{E} \quad 12$ & $1.439 \mathrm{E} \quad 12$ \\
\hline 18500 & $1.943 \mathrm{E} 17$ & $4.741 \mathrm{E} 12$ & $2.902 \mathrm{E} \quad 12$ & $6.909 \mathrm{E} \quad 12$ & $4.463 \mathrm{E} \quad 12$ & $3.862 \mathrm{E} \quad 12$ & $1.299 \mathrm{E} \quad 12$ \\
\hline 19000 & $1.907 \mathrm{E} 17$ & $4.300 \mathrm{E} \quad 12$ & $2.631 \mathrm{E} 12$ & $6.122 \mathrm{E} \quad 12$ & $4.055 \mathrm{E} \quad 12$ & $3.475 \mathrm{E} \quad 12$ & $1.168 \mathrm{E} \quad 12$ \\
\hline 19500 & $1.870 \mathrm{E} \quad 17$ & $3.891 \mathrm{E} \quad 12$ & $2.379 \mathrm{E} \quad 12$ & $5.419 \mathrm{E} 12$ & $3.675 \mathrm{E} \quad 12$ & $3.121 \mathrm{E} 12$ & $1.049 \mathrm{E} 12$ \\
\hline
\end{tabular}

${ }^{*} Z_{0}, Z_{1}$, and $Z_{2}$ are internal partition functions for neutral, singly ionized, and doubly ionized argon atoms respectively. $U_{0}, U_{1}$, and $U_{2}$ are effective ionization energies $\left[\mathrm{cm}^{-1}\right]$.

$N_{e}, N_{0}, N_{1}$, and $N_{2}$ are number densities of electrons, neutral, singly ionized, and doubly ionized argon atoms [cm $\left.{ }^{-3}\right]$ Columns headed by a wavelength contain number densities in the upper state of the line: e.g., under $4300 \mathrm{AI}$ is tabulated $N_{0} g_{u} \exp \left(-E_{u} / k T\right) / Z_{0}$ with $g_{u}=5, E_{u}=116999 \mathrm{~cm}^{-1}$.

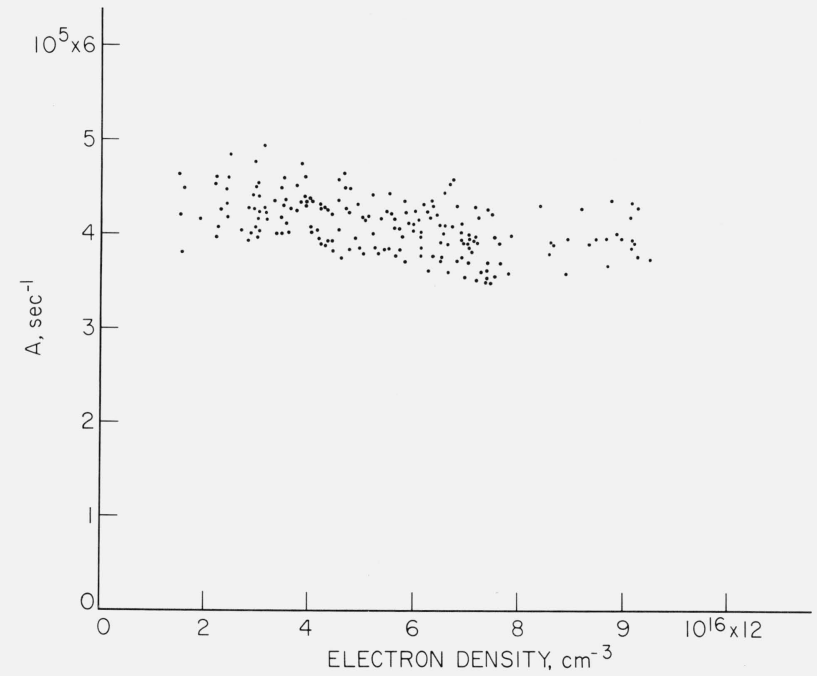

Figure 11. AI 4300 A transition probability values. Ave. $=4.11 \times 10^{5} \mathrm{sec}^{-1}$. Std. dev. $=0.29 \times 10^{5} \mathrm{sec}^{-1}$.

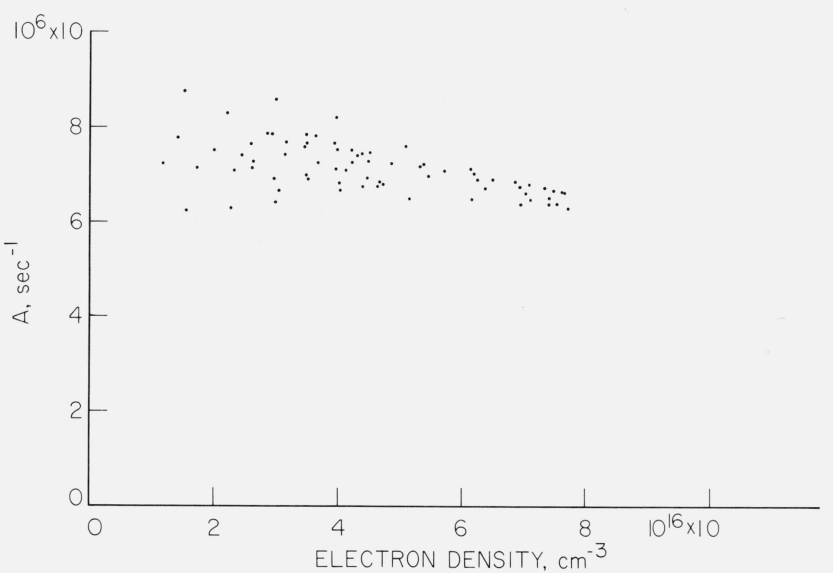

Figure 12. AI 6965 A transition probability values. Ave. $=7.10 \times 10^{6} \mathrm{sec}^{-1}$. Std. dev. $=0.55 \times 10^{6} \mathrm{sec}^{-1}$. obtained from measurements of the AI $6965 \AA$ intensity using a transition probability of $7.10 \times 10^{6}$ $\sec ^{-1}$. The lines in the blue were measured using an analog computer [19] for the direct recording of the Abel inverted spectra. In this way it was felt that the separation of overlapping lines (see fig. 1) could be better accomplished-although somewhat subjectively. The line intensities were determined by planimeter integration and the plasma composition was derived from the measured AI $4300 \AA$ intensity (using $A=4.11 \times 10^{5} \mathrm{sec}^{-1}$ ) for the neutral lines and from the AII $4806 \AA$ intensity (using $A=1.31 \times 10^{8}$ $\sec ^{-1}$ ) for the AII $4348 \AA$ line. Because of the less satisfactory estimates of the line wing corrections, the limited number of experiments, and the uncertainty in the reference transition probabilities the $A$ values for these lines are slightly less accurate than those for the 4300,6965 , and 4806 lines. 


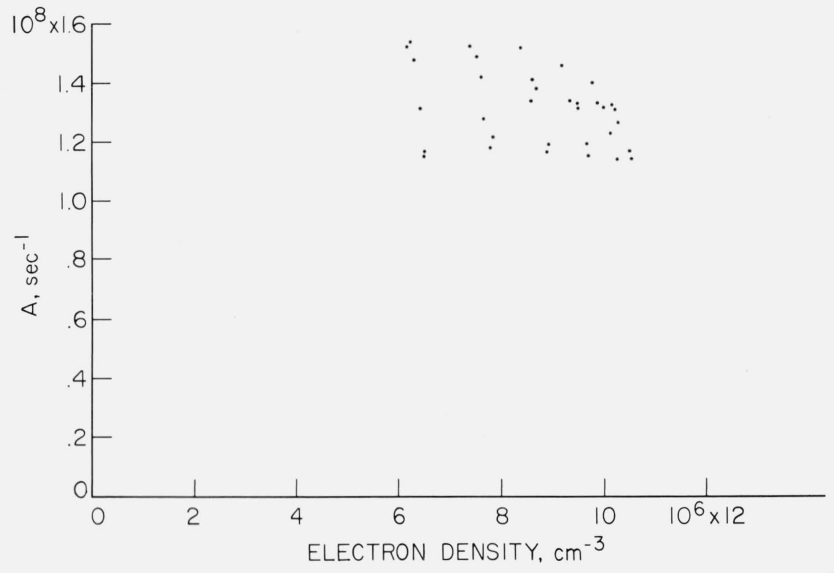

Figure 13. AII $4806 \AA$ transition probability values. Ave. $=1.31 \times 10^{8} \mathrm{sec}^{-1}$. Std. dev. $=0.21 \times 10^{8} \mathrm{sec}^{-1}$

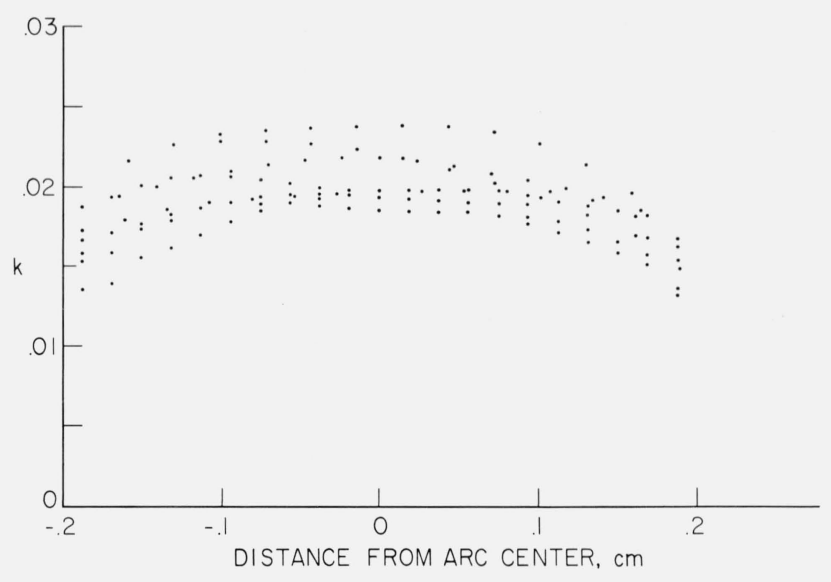

FIGURE 14. The hydrogen/argon atomic ratio, $\mathrm{k}$, as a function of radius for some 60 ampere experiments.

TABle 3. Argon transition probabilities

\begin{tabular}{|c|c|c|c|c|c|c|c|c|}
\hline \multirow{2}{*}{ Wavelength } & \multirow{2}{*}{ Transition } & \multicolumn{7}{|c|}{$A \times 10^{-6}\left[\mathrm{sec}^{-1}\right]$} \\
\hline & & This expt. & Drawin & Gericke & Olsen & Richter & Pery-Thorne & Garstang \\
\hline $\begin{array}{l}4044 \text { I } \\
4159 \text { I } \\
4259 \text { I } \\
4272 \text { I } \\
4300 \text { I } \\
4348 \text { II } \\
4806 \text { II } \\
6753 \text { I } \\
6871 \text { I } \\
6965 \text { I } \\
7030 \text { I } \\
9195 \text { I } \\
9292 \text { I }\end{array}$ & 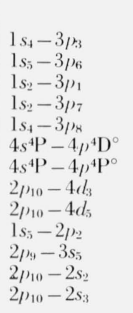 & $\begin{array}{l}0.45 \pm 13 \% \\
1.60 \pm 13 \% \\
4.4 \pm 14 \% \\
0.94 \pm 14 \% \\
0.411 \pm 11 \% \\
240 \pm 27 \% \\
131 \pm 23 \% \\
2.9 \pm 12 \% \\
4.0 \pm 13 \% \\
7.10 \pm 11 \% \\
4.0 \pm 12 \% \\
2.2 \pm 12 \% \\
4.5 \pm 12 \%\end{array}$ & $\begin{array}{l}0.37 \\
1.7 \\
4.0 \\
0.76 \\
.36\end{array}$ & $\begin{array}{c}1.2 \\
3.3 \\
0.69 \\
.32\end{array}$ & $\begin{array}{c}0.66 \\
2.5 \\
0.37 \\
115 \\
79 \\
\\
\\
5.3\end{array}$ & 0.35 & 8.7 & $\begin{array}{r}128 \\
79\end{array}$ \\
\hline
\end{tabular}

\subsection{Hydrogen-Ārgon Ratio}

The ratio of the total hydrogen content to the total argon content as determined from the $\mathrm{H}_{\beta}$ shape and intensity measurements alone is shown in figure 14 as a function of radial position for some $60 \mathrm{~A}$ experiments. This ratio is defined as

$$
k=\left(2 N_{\mathrm{H}_{2}}+N_{\mathrm{H}}+N_{\mathrm{H}+}\right) /\left(N_{\mathrm{A}}+N_{\mathrm{A}+}+N_{\mathrm{A}++}+\ldots\right)
$$

where $N_{\mathrm{H}_{2}}, N_{\mathrm{H}}$, etc., are number densities of the species $\mathrm{H}_{2}, \mathrm{H}$, etc. All of the experiments in figure 14 utilized a premixed tank of the hydrogen-argon mixture with a value for this ratio of about 0.02 as determined by a mass spectrometric analysis. This figure illustrates the "demixing" effect [9] which precludes the use of cold gas composition data in determining the plasma composition in ares containing gas mixtures.

\section{Discussion of Errors}

The recent appearance of a detailed and self-consistent theoretical treatment [9] of the problems of laboratory plasma sources and of spectroscopic plasma diagnostics together with the relatively large data sample sizes that can be accumulated with automatic recording and computing techniques makes possible a much more thorough error analysis than has heretofore been attempted. A discussion of several sources of error follows. In general it is found that the expected experimental and theoretical errors are of roughly comparable magnitudes.

\subsection{Electronic}

The maximum imprecision of the recorded intensities due to instrumental uncertainties was determined to be of the order of 0.15 percent. This figure results from the treatment as independent errors of the maxi- 
mum effects of instrument nonlinearities and zero drift over a period of $1 \mathrm{hr}$ with the combined effects of ambient temperature variations of plus or minus $5{ }^{\circ} \mathrm{C}$ and \pm 10 percent line voltage fluctuations, and includes the contributions from the photomultiplier high-voltage power supply, the current amplifier, the voltage-to-frequency converter and the electronic counter.

An additional variation has been observed in the photomultiplier dark current on the higher gain range settings of the amplifier. This effect became noticeable after the dark current had been suppressed and appeared to consist of a long-term drift of the dark current of as much as 1 percent of full scale accompanied by short-term random noise of a similar magnitude.

The effects of photomultiplier fatigue on the photocurrent measurements were made negligible by restricting anode currents in the photomultiplier to less than $10^{-7} \mathrm{~A}$, a value which previous investigations had shown to be below the threshold of fatigue effects for these tubes.

A certain amount of delay in the response of the photomultipliers to step inputs was observed. A rise time of about $0.2 \mathrm{sec}$ was measured for the phototubes with $\mathrm{S}-20$ response, while the delay was unmeasureable for the $\mathrm{S}-4$ phototubes, implying some dependence on photocathode material. To ensure sufficient time for any effects of photomultiplier delay, mechanical relaxation or transient vibrations to decay, the recording of the photocurrent was postponed until 0.25 seconds after the arc chamber had completed its shift to a new position.

\subsection{Calibration}

The absolute calibration of the source intensities with a tungsten strip lamp is subject to a maximum uncertainty of about \pm 5 percent. The brightness temperature calibration of the strip lamp at NBS is qualified by a certificated uncertainty of $\pm 7{ }^{\circ} \mathrm{C}$ relative to the IPTS which would produce uncertainties of about \pm 3 percent in the transition probabilities of both neutral and ionic lines. For the calculation of the strip lamp emission at wavelengths other than the calibration wavelength $\left(\begin{array}{lll}6530 & \AA\end{array}\right)$ the tungsten emissivities given by DeVos [20] were used. These are estimated to be accurate to within \pm 2 percent. The maximum uncertainty due to this cause would therefore presumably be \pm 2 percent in the blue and infrared spectrum and would decrease to zero at the calibration wavelength.

The errors in intensity due to uncertainties in determination of the correct strip lamp current are around \pm 0.07 percent, almost two orders of magnitude below the errors discussed above and therefore negligible. The combined expected uncertainties are therefore \pm 5 percent for blue and infrared lines decreasing to \pm 3 percent for lines in the red.

\subsection{Arrc Source}

The argon used in these experiments was technical grade argon with typically a purity of 99.996 percent containing mainly nitrogen and oxygen as contaminants. The gas flow system in the arc chamber is designed to prevent atmospheric and electrode contaminants from entering the observation chamber and indeed no impurities were observed spectroscopically during the experiments. From this observation the impurity upper limit and corresponding transition probability error of the order of 1 percent can be set based upon a minimum detectable signal and estimated transition probabilities for lines of the likely impurities, C, N, and 0 .

The arc current regulator maintains the arc current constant to within 0.05 percent for periods of $1 / 4$ or $1 / 2 \mathrm{hr}$. Effects of a long-term drift presumably due to ohmic heating of the oil-cooled regulator shunt were minimized by monitoring the are current at intervals with a potentiometer and precision shunt and making small manual corrections. Oscilloscopic observation of the arc current reveals a 0.3 percent 360 cycle ripple when the rotary converter power supply is used. Assuming the totally unrealistic worst possible case-that the averaging over the 0.1 sec counting time results in a measurement of the electron density at one current extremum and of the line intensity at the other-then the ensuing error in the transition probabilities would be of the order of 0.5 percent and 1 percent for the $\mathrm{AI}$ and $\mathrm{AII}$ lines respectively.

The arc positioning mechanism is capable of locating the arc chamber to within $0.002 \mathrm{~cm}$, with a repeatability an order of magnitude better. Since these positioning errors are attributed to the lead screw and are therefore noncumulative, they will appear on the recorded profile of the traverse as noise amounting at most to about 0.4 percent rms of the maximum signal.

The assumed total arc pressure of $1 \mathrm{~atm}$ is clearly an approximation. No record was kept of the prevailing atmospheric pressure during the experiments so that day-to-day pressure fluctuations of 2 or 3 percent must be expected. The over-pressure of the argon in the arc required to maintain the steady gas flow was $0.0003 \mathrm{~atm}$. The arc column over-pressures due to magnetic self-compression and due to the momentum transport accompanying the heat transport can be estimated [9] for the $90 \mathrm{~A}$ arc as $0.0003 \mathrm{~atm}$ and $0.02 \mathrm{~atm}$ respectively. Thus the actual pressure at all points in the arc was at all times probably within 5 percent of the 1 atm assumed for the calculations. By repeating the plasma composition calculations (table 2) at other pressures the effect of this pressure uncertainty upon the transition probabilities can be evaluated. The result is that a \pm 5 percent error in pressure produces a $\mp 1$ percent error in the neutral line transition probabilities and $\mathrm{a} \pm 8$ percent error in the ionic line values. 


\subsection{Optical}

Any nonuniformity of the arc in the axial direction within the observation chamber will result in transition probability errors due to the finite height of the spectrometer slit which collects light from a region $0.05 \mathrm{~cm}$ long in the axial direction and because of the resulting signal averaging which will be different for line widths, neutral line intensities, and ion line intensities. Estimates of the maximum errors from this source based upon measurements taken during a crude scan along the arc axis are 0.5 percent and 4 percent for neutral and ionic line transition probabilities respectively. Similar errors arise in the steep gradient regions of the arc because of imaging errors of the optical system and the finite width of the entrance slit. The combined lateral image smearing was determined to be less than $0.005 \mathrm{~cm}$ for the $15 \mu$ slit by moving the edge of a tungsten strip filament lamp across the optic axis at the arc position and observing the "halfwidth" of the signal step. The corresponding maximum transition probability errors in this case are also 0.5 percent and 4 percent respectively at the edges of the arc. In all cases the experimental values should be too high. Turbulence, if present in the arc, would lead to similar errors. However, estimates of the Reynolds number for the conditions of these experiments lie well within the laminar flow region suggesting that this should not be a problem. Optical refractivity gradients in the arc are expected to cause a negligible distortion of the arc intensity profile even in the vicinity of strong lines [9]. This expectation was partially confirmed experimentally by the failure to detect a significant displacement of the apparent position of the edge of a tungsten strip lamp filament imaged into the arc plasma at any observing wavelength near the AI $6965 \AA$ line.

Scattering of light by the optical elements and arc chamber windows would cause an increase in the apparent transition probabilities at the higher radii, i.e., at the edges of the luminous arc column. Since a slight variation of this type was indeed observed, several experiments were performed with no window in the arc chamber. These failed to show a significant difference at the arc edges from those in which the window was included in the optical path. Direct rough measurements of the light scattered by the optical elements showed this amount to be less than 0.3 percent of the maximum source intensity, which could not possibly account for the observed radial variation of the $A$-values.

The shot noise arising from statistical fluctuation in the arrival of photons at the photomultiplier can be estimated from the source intensity and the geometry of the optical train. It is estimated to be always less than 0.5 percent for the strip lamp calibration and less than 0.1 percent for the arc, except at the extreme edges.

For some experiments the limiting aperture of the optical system was reduced to one-half its original size. Any observed difference in the results of these experiments would have pointed out errors and misalinements of the optical system. No such differences were observed, indicating that the optical system was properly alined and that the original aperture was indeed limiting.

The degree of self-absorption can be estimated by calculating the optical depth $\tau=2 \int_{0}^{R} \kappa^{\prime} d r$

where $\quad \kappa^{\prime}=\epsilon / B(T)$

and

$$
B(T)=2 h c^{2} \lambda^{-5}\left(e^{h c / \lambda k T}-1\right)^{-1}
$$

$\kappa^{\prime}$ is the effective absorption coefficient $\left(\mathrm{cm}^{-1}\right)$, and $\epsilon$ is the observed radially resolved emission coefficient per steradian (erg sec$\left.-1 \mathrm{~cm}^{-3} \mathrm{sr}^{-1} \mathrm{~cm}^{-1}\right)$. The results of such calculations at the centers of the three lines at 4300,6965 , and $4806 \AA$ are respectively $0.002,0.11$, and 0.002 for the optical depths, $\tau$, in the worst cases encountered. Since the Abel inversion is most unstable near the center due to the infinite kernel at that point it is to be expected that the consequences of errors in the side-on intensity measurements due to self-absorption will normally be most severe in the radially inverted values near the center of the arc. This is borne out by exact analytic calculations of simple models and by the approximate Freeman and Katz [10] treatment of more realistic models. These calculations indicate that for small degrees of selfabsorption and for bell-shaped profiles the relative error in the radially resolved emission coefficient is of the order of $\kappa_{0}^{\prime} R$ at the arc center where $\kappa_{0}^{\prime}$ is the effective absorption coefficient at the center and $R$ is, as before, the nominal arc radius. Thus only in the case of the AI $6965 \AA$ line will self-absorption play a role leading to transition probabilities that are too low by about 5 percent ( $\kappa_{0}^{\prime} R$ averaged over the line shape) at the arc center and increasing to the correct value at the edge. To some extent this conclusion appears to be supported by the data of figures 11 and 12 which show for the $6965 \AA$ line a more pronounced depression of the transition probabilities at high electron densities near the arc center.

\subsection{Analytical and Theoretical}

In general, the accuracy of an Abel inversion based on curve fitting techniques is dependent upon the form of the intensity profile of the arc traverse and the fitting function, the number of polynomials used in the fitting function, the number of increments examined during each arc traverse, and the noise present on the intensity profile. To assess the effects of these variables on the accuracy of the inversion, analytical test functions were constructed with varying amounts of superimposed noise drawn from a table of random normal deviates. These test functions, which are of the same general shape as the observed profile of the arc traverse, were then inverted by the machine program used in processing the experimental results. As expected, for data with a relatively high noise level a small number of polynomials in the fitting function produces the 
best inversion in terms of accuracy of fit and minimum noise amplification. Conversely, no significant improvement in accuracy results from using large numbers of increments and terms unless the signal/noise ratio of the data is quite high. The accuracy of the inversion is, however, relatively insensitive to variations in number of increments for noise levels greater than 0.3 percent due to the smoothing inherent in the curve fitting process and in this range the relative noise level of the inverted profile is almost identical to that on the original recorded intensity profile.

The results of tests on analytical functions were borne out by further tests of the inversion on actual experimental profiles. The number of data points of representative many-point arc traverses was halved and quartered by systematically discarding alternate data points, and the resulting "thinned" traverses inverted and compared to the originals. These tests also showed that, at least for the data encountered here, no further accuracy could be attained by taking more than 20 or 30 measurements per arc traverse. Similarly, the use of more than 8 or 10 terms in the polynomial expansion was again not warranted and, in fact, increased the noise level in the results.

Numerical tests of the dispersion shape fitting program carried out by superimposing noise upon calculated dispersion shapes reveals that the relative rms noise on the computed line intensity and halfwidth is roughly equal to the relative noise in the input intensity data at the half maximum intensity level. Thus, little smoothing of the noise is accomplished in this step. This is due mainly to the influence of the noise upon the background or continuum level determination by the program. In these experiments this determination was dependent upon a comparatively small effective number of points due to the wavelength point distribution employed.

The use of the area of the fitted dispersion shape as the spectral line intensity obviates the necessity for correcting measured line intensities for that portion of the line lying below and beyond the end points of the wavelength scan. This is a significant correction amounting to 13 percent for scans of only ten halfwidths and may account for part of the difference between the transition probabilities observed here and the lower values of other workers. Since the experimental line shapes even after correction for apparatus functions are not expected to be quite dispersion shapes [9] it was necessary to ascertain the effect of departures from the dispersion shape upon the linewidth, shift, and intensity values as measured in these experiments. For this purpose the dispersion shape fitting program was applied to a theoretical complete asymmetric line shape [21] which appears to be reasonably representative of the experimental line shapes encountered here. The computed intensities and halfwidths were consistently low by 3 percent and 2 percent respectively. The line shifts were consistently overestimated by a magnitude approximately equal to the error in the $\frac{1}{2}$ halfwidth. No attempt was made to calibrate the dispersion shape fitting program for these theoretical line shapes because of the unavailability of sufficiently extensive tables of the necessary line shapes. Consequently, systematic errors can be expected in the results amounting to perhaps 5 percent and probably always in the direction indicated by this example.

At the heart of these measurements is the determination of the electron density by the $\mathrm{H}_{\beta}$ line shape measurement. Unfortunately a significant uncertainty in the results is associated with this step. For example, Griem [9] estimates that the electron densities obtained from $\mathrm{H}_{\beta}$ line shapes are probably reliable only to about 5 percent based upon an analysis of the theoretical approximations. In addition, as indicated in figure 4, some error is involved in the fitting of a theoretical line shape to the experimental data. Typically the rms deviation of the experimental points from the fitted curve is 2 or 3 percent of the maximum $\mathrm{H}_{\beta}$ intensity. (This is somewhat better than the dispersion shape fitting to the argon lines which typically has an rms deviation of 3 to 5 percent. However, in part this greater error is explained by the influence of the apparatus or slit function which is not taken into account in the fitting and by the approximation of the true theoretical shapes by the dispersion shape.) The subtraction of the pure argon spectrum from the argon +hydrogen spectrum removes the major part of any uncertainties in the $\mathrm{H}_{\beta}$ shape measurement due to irregularities in the argon continuum underlying the hydrogen line. Any errors in this step due to small changes in the radial temperature profile caused by the addition of the trace of hydrogen are expected to be negligible but presumably are included in this 2 or 3 percent deviation. As a partial check of these $\mathrm{H}_{\beta}$ measurements in two of the $60 \mathrm{~A}$ experiments $\mathrm{H}_{\alpha}$ line shapes were also recorded. From the electron density, temperature, and hydrogen to argon ratio as determined from the $\mathrm{H}_{\beta}$ measurements the theoretical intensity and shape of the $\mathrm{H}_{\alpha}$ lines were computed using the Griem, Kolb, and Shen tabulations. Figure 15 shows a comparison of an experimental $\mathrm{H}_{\alpha}$ line shape (after the Abel inversion and subtraction of the pure argon spectrum) with the theoretical shape based upon the $\mathrm{H}_{\beta}$ measurements at the same radial point. The agreement is seen to be satisfactory in the wings and at the center but the halfwidth of the theoretical shape is 10 percent or so smaller than the experimental one. This difference appears to be in accord both in magnitude and direction with the correction to the theoretical $\mathrm{H}_{\alpha}$ shapes which Griem et al., [14] estimate would accrue by taking into account the improved ion field strength distribution functions of Mozer and Baranger. Thus, in conclusion, it is reasonable to assume that the electron densities obtained in these experiments from the $\mathrm{H}_{\beta}$ line shapes should not be in error by much more than about 5 percent. This results in 8 and 20 percent uncertainties respectively in the AI and AII transition probabilities.

Griem [22] has discussed in detail the validity of the local thermodynamic equilibrium (L.T.E.) assumption in laboratory plasmas. Using his criteria one finds 


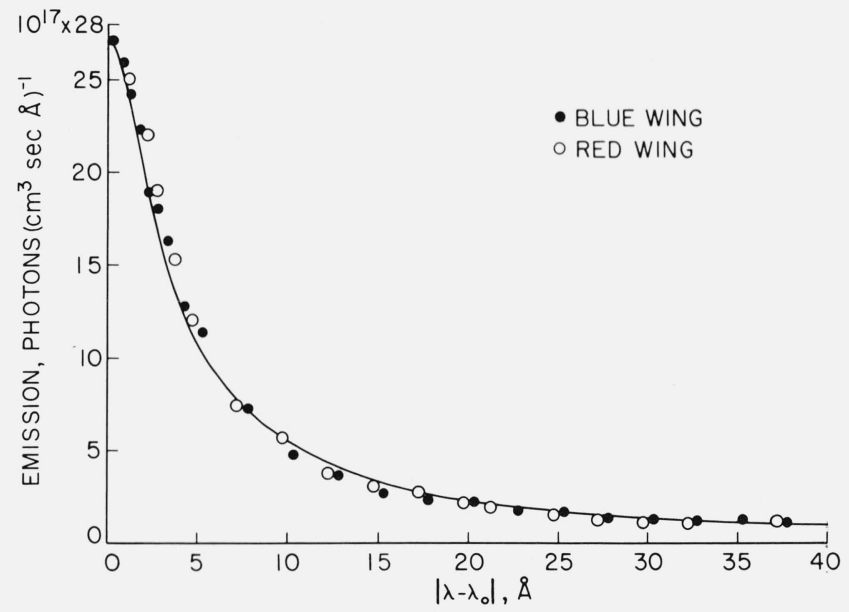

FIGURE 15 Experimental intensity points in the neighborhood of $\mathrm{H}_{\alpha} 6563 \AA$ after Abel inversion (on axis of 60 ampere arc in argon with $\sim 1 \% \mathrm{H}_{2}$ ) with theoretical $\mathrm{H}_{\alpha}$ line shape computed from parameters obtained by $\mathrm{H}_{\beta}$ measurements: $\mathrm{N}_{\mathrm{e}}=7.35 \times 10^{16} \mathrm{~cm}^{-3}$, $\mathrm{k}=0.0197$.

that for homogeneous plasmas of the dimensions of the arcs of these experiments complete L.T.E. would be probable down to the lowest electron densities actually utilized in the measurements $-2 \times 10^{16}$ for neutral species and $5 \times 10^{16}$ for ionic species. Again following Griem's treatment one finds that the greatest average distance traveled by any species in the arc between equilibrating collisions is that of the ground state neutrals of the order of $10^{-2} \mathrm{~cm}$ at the axis. Thus, the measured electron density and line intensities at a particular point in the arc are not to be interpreted as corresponding exactly to L.T.E. conditions at the local electron temperature at that point but rather as appropriate to some kind of averaging of near-equilibrium distributions which exist over a distance of the order of $10^{-2} \mathrm{~cm}$. The use of this distance, then, permits some estimate of the effects of the temperature and composition gradients present in the arc upon the accuracy of the transition probability determinations. Clearly an over-estimate of the errors is obtained by coupling line intensity measurements at one point with equilibrium calculations based upon electron density measurements $10^{-2} \mathrm{~cm}$ away. Such a procedure result 3 in error estimates of 1 percent for the neutral lines and 2 percent for the ionic lines at the center of the $90 \mathrm{~A}$ arc. Away from the center this equilibrating collision distance is not a proper measure of the L.T.E. smearing because of the additional equilibrating influences of the diffusion of ions and excited atoms from the hot core and the absorption of resonance radiation from the core. The fact that the measured transition probabilities show no strong dependence upon the electron density, however, suggests that if departures from L.T.E. are not serious at the arc center they are probably also not serious at least out to $0.2 \mathrm{~cm}$ where the lowest electron density measurements were taken.

\section{Discussion of Results}

The estimated transition probability uncertainties resulting from this error analysis assuming the errors are independent are included in table 3 together with transition probability values obtained by other investigators. Most of the errors which have been discussed are systematic. However, a few-the electronic noise, the numerical curve fitting errors, the day-today pressure variation, and the dark current drift errors - are not and will tend to be reduced in magnitude by the averaging over the many experiments or radii. In arriving at the uncertainty estimates in table 3 due allowance for this kind of error was taken, where appropriate, by division by the square root of the sample number. A similar calculation applied to the neutral line width and shift measurements gives an estimated 6 percent uncertainty.

The three dominating errors in the average transition probability values determined here are the absolute intensity calibration errors, the systematic errors in the argon line shape fitting due to apparatus functions and asymmetry, and the estimated uncertainty in the hydrogen line broadening theory. It is now possible to reduce the effect of calibration uncertainties from the 3 to 5 percent estimated in these experiments to about 1 to 2 percent, by means of new NBS radiometric calibration services which have become available on a limited basis [24] since these experiments were undertaken. Also, with some effort, the argon line shape fitting can be improved by the calculation and use of more extensive theoretical line shape tables including asymmetry and possibly with apparatus and Doppler broadening properly folded in. Thus the experimental errors can probably be reduced by a factor of two without excessive effort. However, without a concomitant improvement in the accuracy of the electron density determination these experimental improvements would only marginally reduce the estimated overall errors. The uncertainty in this step is the major contribution to the error estimates in table 3. Griem's estimate of the accuracy of the hydrogen line shape calculation is not significantly changed by consideration of the small amount of relatively contradictory experimental evidence [9]. Further experimental or theoretical studies should permit a better assessment of the accuracy of this extremely useful plasma diagnostic technique.

The origin of the apparent decrease of the transition probabilities with increasing electron density (see e.g., fig. 11) is unknown. Of the error sources described above, the only pertinent ones estimated to be of sufficient magnitude to encompass this effect are hydrogen line broadening theory errors, line shape fitting errors, and possibly departures from L.T.E. This deviation from a constant transition probability agrees qualitatively with the discrepancies observed by Wiese et al. [25] in pure hydrogen arcs.

The scatter of the transition probability values (figs. 4 to 13 ) is in agreement with the noise level estimates described above. These were mainly electronic and 
amounted to 1 or 2 percent of the maximum signals. When expressed in terms of the half-intensity of a line this relative noise level is higher by a factor of two or more, and, consequently, since neither the Abel inversion nor the dispersion line fitting seems to modify the noise level, the line intensities should exhibit a scatter of several percent. When combined with a similar noise in the electron density values a resultant scatter of the transition probability values of the order of 5 to 10 percent is to be expected.

The theoretical transition probabilities of Garstang [23], the "hook" method values of Pery-Thorne and Chamberlain [1], and the arc measurements of Drawin [4] appear to agree generally with the present values in the sense that the error ranges assigned by the investigators overlap the present ones. (The theoretical values are good to perhaps a factor of two, PeryThorne and Chamberlain estimate an accuracy of 20 to 30 percent for their values, and Drawin assigns error limits ranging from 10 percent for AI 4044 A to 20 percent for AI $4300 \AA$.) Drawin's measurements were carried out in an arc containing a hydrogen-argon mixture. However, since his diagnostics were based upon hydrogen line intensity measurements and the use of the cold gas composition there is a possibility of a systematic error due to demixing.

Gericke's argon transition probabilities [3] are based upon an argon arc temperature profile derived from measurements of the AII 4348 A line intensity using a transition probability value of $68 \times 10^{6} \mathrm{sec}^{-1}$. The value obtained in the present work for this transition probability is $240 \times 10^{6} \mathrm{sec}^{-1}$. If this is correct then Gericke's transition probability values should be increased by about 35 percent ${ }^{3}$ and would agree well with the present values. These same comments apply to Richter's value for the AI 4044 A line (27) which was evidently obtained similarly. The corresponding adjustments to Gericke's electron density values result, in the case of the AI $4300 \AA$ line, in values (in $\AA$ ) for the $\frac{1}{2}$-halfwidth and shift both approximated by 0.12 $\times 10^{-16}\left[N_{e}\right]$ where $N_{e}$ is the number of electrons per $\mathrm{cm}^{3}$. This compares reasonably well with the present values (table 1 ) of $0.10 \times 10^{-16}\left[N_{e}\right]$. Thus the measurements reported here are evidently well in accord with Gericke's on a relative basis but the absolute results differ through the use of the $\mathrm{H}_{\beta}$ line shape for the arc diagnostics rather than the AII $4348 \AA$ transition probability used by Gericke.

In the case of Olsen's results an estimate of the degree of self-absorption at the center of the AII $4806 \AA$ line $^{4}$ under his arc conditions leads to values for the optical depth, $\tau$, of 0.7 or 0.3 depending upon whether the line halfwidth is estimated from Griem's theoretical calculations [9] or is extrapolated from the experimental data of figure 9. In either case the magnitude is sufficient to cause a serious error in the radially resolved emission coefficient and to raise doubts about whether Olsen's observed apparent

\footnotetext{
3 This includes a correction discussed by Griem (26) to the ionization potential lowering. ${ }^{4}$ There appears to be an error in the equation for self-absorption in reference 2 (eq (6)). $g_{n}$ should probably be replaced by $\frac{1}{2} \pi$.
}

off-axis maximum was not in fact a consequence of this absorption rather than a manifestation of the Larenz and Bartels [28] "normal" temperature effect. In this event Olsen's temperature determinations and transition probabilities are open to radical reinterpretation.

Table 1 compares the measured values of the line widths and shifts (averaged assuming they are proportional to the electron density) with theoretical values computed from Griem's tables [9] for a temperature of $12000{ }^{\circ} \mathrm{K}$. Fortunately the theoretical values of the reduced widths and shifts are not strongly temperature dependent so that the difference between $12000^{\circ}$ and whatever effective temperature is introduced by the averaging of the experimental values will cause a difference of only a few percent in the tabular entries. For the neutral argon lines the agreement of theory and experiment is roughly within the expected accuracy of 8 percent for the experiments and 20 percent for the theory. The ion line results, however, are in very poor agreement with theory. For the line shift this is understandable since the theoretical Stark shift for the line does not include the plasma polarization blue shift [9] which rough calculations indicate will be larger than the Stark shift. For the width of the AII $4806 \AA$ line errors due to both Doppler broadening and the slit width correction procedure are important but do not appear to be sufficient to resolve the discrepancy between theory and experiment. The consequences of neglecting the Doppler broadening were estimated by folding the Doppler line shape (Gaussian with $\frac{1}{2}$-halfwidth of approximately $0.03 \AA$ ) into the apparatus function to give a broadened effective apparatus function. The use of this modified apparatus function (which significantly affects only the narrow ionic line) reduces the widths of figure 9 by approximately $0.005 \AA$ and decreases the proportionality constant in table 1 by about 5 percent. The error in this proportionality constant stemming from the application of the slit width correction procedure to the AII $4806 \AA$ line where the line and apparatus function widths are comparable (line width: $0.08 \AA$ to $0.15 \AA$ : apparatus width: $0.1 \AA$ ) is estimated at 10 or 20 percent. This figure is based upon the results of the $150 \mu$ slit width experiment in which the apparatus function was wider than the AI $4300 \AA$ line at all radii. In this case application of the slit width correction procedure to the computer fitted line widths resulted in an average value for $\frac{w}{N_{e}} \times 10^{16}$ of 0.089 compared to the average 0.100 for all the narrow slit experiments.

\section{References}

[1] A. Pery-Thorne and J. E. Chamberlain. Proc. Phys. Soc. (London) 82, 133 (1963).

[2] H. N. Olsen, J. Quant. Spectr. Radiative Transfer 3, 59 (1963).

[3] W. E. Gericke, Z. Astrophysik 53, 68 (1961).

[4] H. W. Drawin, Z. Physik 146, 295 (1956).

[5] W. L. Wiese and J. B. Shumaker, Jr., J. Opt. Soc. Am. 5 1, 937 (1961). 
[6] J. B. Shumaker, Jr., Rev. Sci. Instr. 32, 65 (1961).

[7] C. R. Yokley and J. B. Shumaker, Jr., Rev. Sci. Instr. 32, 6 (1961).

[8] D. R. Paquette and W. L. Wiese, Applied Optics 3, 291 (1964).

[9] H. R. Griem, Plasma Spectroscopy (McGraw-Hill Book Co., New York, N.Y., 1964).

[10] M. P. Freeman and S. Katz. J. Opt. Soc. Am. 50, 826 (1960).

[11] M. P. Freeman and S. Katz, J. Opt. Soc. Am. 53, 1172 (1963).

[12] A. Erdélyi, Higher Transcendental Functions, Vol. 2 (McGrawHill Book Co., New York, N.Y., 1953).

[13] S. I. Herlitz. Arkiv för Fysik. 23, 571 (1963).

[14] H. R. Griem, A. C. Kolb, and K. Y. Shen, Astrophys. J. 135, 272 (1962).

[15] H. R. Griem, Astrophys. J. 136, 422 (1962).

[16] J. B. Shumaker, Jr., and W. L. Wiese, Temperature-Its Measurement and Control in Science and Industry, Vol. 3, Pt. 1, pp. 575-579, (Reinhold Pub. Corp., New York, N.Y., 1962).

[17] K. S. Drellishak, C. F. Knopp, and A. B. Cambel, Arnold Engineering Development Center Tech. Documentary Report AED C-TDR-63-146 (1962).
[18] H. R. Griem, Phys. Rev. 128, 997 (1962).

[19] C. R. Yokley and J. B. Shumaker, Rev. Sci., Instr. 34, 551 (1963).

[20] J. C. DeVos, Physica 20, 690 (1954)

[21] O. Roder and A. Stampa, Z. Physik 1 78, 348 (1964).

[22] H. R. Griem, Phys. Rev. 131, 1170 (1963).

[23 R. H. Garstang, Mon. Not. R. Astr. Soc 114,118 (1954).

[23] N.B.S. Tech. News Bull. 49, 14 (1965).

[25] W. L. Wiese, D. R. Paquette, and J. E. Solarski, Phys. Rev. 129, 1225 (1963).

[26] H. R. Griem, Phys, Rev. 128, 515 (1962).

[27] J. Richter, Z. Astrophysik 53, 262 (1961).

[28] R. W. Larenz and H. Bartels, Naturwiss. 37, 164 (1950).

(Paper 69A6-370) 